\title{
The rapid convergence for nonlinear singular differential systems with "maxima"
}

\author{
Peiguang Wang ${ }^{\mathrm{a}, *}$, Xiang Liu ${ }^{\mathrm{a}}$, Tongxing $\mathrm{Li}^{\mathrm{b}}$ \\ ${ }^{a}$ College of Mathematics and Information Science, Hebei University, Baoding, Hebei 071002, China. \\ ${ }^{b}$ School of Information Science and Engineering, Linyi University, Linyi, Shandong 276005, China.
}

Communicated by M. Bohner

\begin{abstract}
This paper investigates the initial value problem for a class of nonlinear singular differential systems with "maxima". By using the comparison principle and the approximate quasilinearization method, we obtain two monotone iterative sequences of approximate solutions which converge uniformly and rapidly to the solution of such systems. (c)2017 All rights reserved.
\end{abstract}

Keywords: Singular system, maxima, approximate quasilinearization, rapid convergence. 2010 MSC: 34A45, 34K07.

\section{Introduction}

It is well-known that singular differential systems can be used to model many problems, such as nonNewtonian fluid mechanics, optimal control problems, and electrical circuits. The concept of singular systems was introduced by Rosenbrock [20] in 1974, it is more complicated than the ordinary ones, and its qualitative analyses involve greater difficulty than those of the ordinary systems. Therefore researching the theory of singular differential systems has profound significance. Previous studies have mainly focused on the linear and nonlinear singular systems described by ordinary differential equations. The basic theory of the linear singular systems can be found in [10,11]. Up till now, the theory of nonlinear singular systems is imperfect, in which the convergence of the solution is one of the most concerned problems, which has an important effect on the qualitative theory development.

Quasilinearization is a very efficient method to obtain approximate solutions of nonlinear problems. This method originated in the dynamic programming theory and was initially applied by Bellman and Kalaba [8]. A systematic development of the method to ordinary differential equations has been provided by Lakshmikantham and Vatsala [18], and there are some generalized results of the method to various types of differential systems, we can refer to the monographs [16, 17], for functional differential equations $[4,12]$, for impulsive equations [3, 6], for partial differential equations [5, 9, 14, 24], for others [21, 22,

\footnotetext{
${ }^{*}$ Corresponding author Li)

Email addresses: pgwang@hbu.edu.cn (Peiguang Wang), 1065265114@qq.com (Xiang Liu), litongx2007@163.com (Tongxing

doi:10.22436/jnsa.010.10.25
}

Received 2017-08-12 
27] and references cited therein. However, there were few applicable results of the method to singular differential systems $[1,13,19,23]$.

Differential equations with "maxim" are a special type of differential equations that contain the maximum of the unknown function over a previous interval(s). Recently, the interest in differential equations with "maxim" has increased exponentially (see Bainov and Hristova [7]). By using the method of quasilinearization, Agarwal and Hristova [2] and Hristova et al. [15] investigated the initial value problem for differential equations with "maxim" and difference equations with "maxim", respectively. Wang and Liu $[25,26]$ obtained the convergence uniformly and quadratically to singular differential systems with "maxim" and singular difference systems with "maxim". In this paper, we attempt to extend the applications of approximate quasilinearization method, and study the rapid convergence of the solution for a class of nonlinear singular differential system with "maxim". The main work focuses on two aspects: one is to construct the monotone sequences for such systems, which is a Taylor series numerical method in which the truncation is chosen so that the iterates is of order $k$; the other is to prove the convergence uniformly and rapidly to the solution of the problem.

\section{Preliminaries}

Consider the following initial value problem for the singular differential system with "maxim" (IVP)

$$
\left\{\begin{array}{l}
A x^{\prime}(t)=f\left(t, x(t), \max _{s \in[t-h, t]} x(s)\right), t \in[0, T], \\
x(t)=\varphi(t), t \in[-h, 0],
\end{array}\right.
$$

where $A$ is a singular $n \times n$ matrix, $x \in R^{n}, f \in C\left([0, T] \times R^{n} \times R^{n}, R^{n}\right), \varphi \in C\left([-h, 0], R^{n}\right)$, and $h$ and $T$ are fixed positive constants.

Definition 2.1. The function $\alpha_{0} \in C^{0}\left([-h, T], R^{\mathfrak{n}}\right) \cup C^{1}\left([0, T], R^{\mathfrak{n}}\right)$ is called a lower (an upper) solution of IVP (2.1), if the following inequalities are satisfied:

$$
\left\{\begin{array}{l}
A \alpha_{0}^{\prime}(t) \leqslant(\geqslant) f\left(t, \alpha_{0}(t), \max _{s \in[t-h, t]} \alpha_{0}(s)\right), \quad t \in[0, T], \\
\alpha_{0}(t) \leqslant(\geqslant) \varphi(t), \quad t \in[-h, 0] .
\end{array}\right.
$$

Let the functions $\alpha_{0}, \beta_{0} \in C\left([-h, T], R^{n}\right)$ be such that $\alpha_{0}(t) \leqslant \beta_{0}(t)$. Define the following sets for convenience.

$$
\begin{aligned}
S\left(\alpha_{0}, \beta_{0}\right) & =\left\{u \in C\left([-h, T], R^{n}\right) \mid \alpha_{0}(t) \leqslant u(t) \leqslant \beta_{0}(t), t \in[-h, T]\right\}, \\
\Omega\left(\alpha_{0}, \beta_{0}\right) & =\left\{(t, x, y) \in[0, T] \times R^{n} \times R^{n} \mid \alpha_{0}(t) \leqslant x(t) \leqslant \beta_{0}(t), \max _{s \in[t-h, t]} \alpha_{0}(s) \leqslant y(t) \leqslant \max _{s \in[t-h, t]} \beta_{0}(s)\right\} .
\end{aligned}
$$

In our further investigations, we need some results on linear singular differential inequalities and systems and thus for the convenience of readers, we present some relevant results here.

Consider the singular differential inequalities

$$
A x^{\prime}(t)+M(t) x(t) \leqslant 0, x(0) \leqslant 0, \quad t \in[0, T],
$$

where $A, M(t)$ are $n \times n$ matrices, $A$ is singular and $M(t)$ is continuous on $[0, T]$.

Lemma 2.2. Assume that

$\left(\mathrm{H}_{2.1}\right)$ there exists a constant $\lambda$ such that, $\mathrm{L}(\mathrm{t})=[\lambda \mathrm{A}+\mathrm{M}(\mathrm{t})]^{-1}$ exists and $\hat{\mathrm{A}}=\mathrm{AL}(\mathrm{t})$ is a constant matrix; $\left(\mathrm{H}_{2.2}\right)$ there exists a nonsingular matrix $\mathrm{Q}$ such that $\mathrm{Q}^{-1},(\mathrm{LQ})^{-1}$ exist and $\mathrm{Q}^{-1},(\mathrm{LQ}),(\mathrm{LQ})^{-1} \geqslant 0$, satisfying

$$
\mathrm{Q}^{-1} \hat{\mathrm{A}} \mathrm{Q}=\left(\begin{array}{ll}
\mathrm{C} & 0 \\
0 & 0
\end{array}\right), \mathrm{Q}^{-1}[\mathrm{I}-\lambda \hat{\mathrm{A}}] \mathrm{Q}=\left(\begin{array}{cc}
\mathrm{I}_{1}-\lambda \mathrm{C} & 0 \\
0 & \mathrm{I}_{2}
\end{array}\right),
$$

where $\mathrm{C}$ is a diagonal matrix with $\mathrm{C}^{-1} \geqslant 0$.

Then $x(0) \leqslant 0$ implies $x(t) \leqslant 0$ on $[0, T]$. 
For the singular linear initial value problem

$$
A x^{\prime}(t)+M(t) x(t)=g(t), \quad x(0)=x_{0},
$$

we have the following known result.

Lemma 2.3 ([11]). Assume that the condition $\left(\mathrm{H}_{2.1}\right)$ of Lemma 2.2 holds, index $(\mathrm{A})=1$, and $\left(\mathrm{H}_{2.3}\right)$ yo satisfies $\left(\mathrm{I}-\hat{\mathrm{A}} \hat{A}^{\mathrm{D}}\right)\left(\mathrm{y}_{0}-w(0)\right)=0$, where $w(\mathrm{t})=\hat{M}^{\mathrm{D}} \mathrm{g}(\mathrm{t}), \hat{M}=\mathrm{M}(\mathrm{t}) \mathrm{L}(\mathrm{t})$.

Then the unique solution $\mathrm{y}(\mathrm{t})$ of

$$
\hat{A} y^{\prime}(t)+\hat{M} y(t)=g(t), y(0)=y_{0},
$$

is given by

$$
y(t)=e^{-\hat{A}^{D} \hat{M} t} \hat{A} \hat{A}^{D} y_{0}+e^{-\hat{A}^{D} \hat{M} t} \int_{0}^{t} e^{\hat{A}^{D} \hat{M} \sigma} \hat{A}^{D} g(\sigma) d \sigma+\left(I-\hat{A} \hat{A}^{D}\right) \hat{M}^{D} g(t),
$$

where $\hat{A}^{\mathrm{D}}$ and $\hat{M}^{\mathrm{D}}$ denote the Drazin inverse of the matrices $\hat{\mathrm{A}}$ and $\hat{\mathrm{M}}$, respectively. We note that once we have known $\mathrm{y}(\mathrm{t})$, then we get $\mathrm{x}(\mathrm{t})=\mathrm{L}(\mathrm{t}) \mathrm{y}(\mathrm{t})$, where $\mathrm{x}(\mathrm{t})$ is the solution of (2.2).

We will prove the following comparison result which plays an important role for the establishment of our main results.

Consider the singular differential inequalities

$$
\left\{\begin{array}{l}
A x^{\prime}(t)+M(t) x(t)+N(t) \max _{s \in[t-h, t]} x(s) \leqslant 0, t \in[0, T], \\
x(t) \leqslant 0, \quad t \in[-h, 0],
\end{array}\right.
$$

where $A, M(t)$ are $n \times n$ matrices, $A$ is singular, and $M(t), N(t)$ are continuous on $[0, T]$.

Lemma 2.4. Assume that the conditions $\left(\mathrm{H}_{2.1}\right)-\left(\mathrm{H}_{2.3}\right)$ hold, and

$\left(\mathrm{H}_{2.4}\right)$ The matrix $[\mathrm{I}-\mathrm{M}]^{-1}$ exists and is nonnegative, and $\mathrm{N}(\mathrm{t}) \leqslant 0$ for $\mathrm{t} \in[0, \mathrm{~T}]$, where

$$
M=\max _{s \in[0, T]}\left\{-[\lambda A+M(s)]^{-1}\left[e^{-\hat{A}^{D} \hat{M} s} \int_{0}^{s} e^{\hat{A}^{D} \hat{M} \sigma} \hat{A}^{D} N(\sigma) d \sigma+\left(I-\hat{A} \hat{A}^{D}\right) \hat{M}^{D} N(s)\right]\right\} .
$$

Then $x(t) \leqslant 0$ on $[-h, T]$.

Proof. In view of the assumption $\mathrm{N}(\mathrm{t}) \leqslant 0$, we can get from $(2.3)$ that

$$
A x^{\prime}(t)+M(t) x(t) \leqslant-N(t) \max _{s \in[-h, T]} x(s), \quad t \in[0, T], \quad x(t) \leqslant 0, \quad t \in[-h, 0] .
$$

Now, we consider the following two cases:

Case 1. If $\max _{s \in[0, T]} x(s) \leqslant \max _{s \in[-h, 0]} x(s)$, then we have $\max _{s \in[-h, T]} x(s)=\max _{s \in[-h, 0]} x(s)$, and according to the assumption that $x(t) \leqslant 0$ on $[-h, 0]$, the inequalities (2.4) can be written in the form

$$
A x^{\prime}(t)+M(t) x(t) \leqslant-N(t) \max _{s \in[-h, 0]} x(s) \leqslant 0, \quad t \in[0, T], \quad x(t) \leqslant 0, \quad t \in[-h, 0] .
$$

Noting that $x(0) \leqslant 0$, applying Lemma 2.2 , we get $x(t) \leqslant 0$ on $[0, T]$.

Case 2. If $\max _{s \in[-h, 0]} x(s) \leqslant \max _{s \in[0, T]} x(s)$, then we have $\max _{s \in[-h, T]} x(s)=\max _{s \in[0, T]} x(s)$, the inequalities (2.4) can be written in the form

$$
A x^{\prime}(t)+M(t) x(t) \leqslant-N(t) \max _{s \in[0, T]} x(s), \quad t \in[0, T], \quad x(t) \leqslant 0, \quad t \in[-h, 0] .
$$

Lemma 2.2 shows that $x(t) \leqslant y(t)$ on $[0, T]$, where $y(t)$ is the solution of 


$$
A y^{\prime}(t)+M(t) y(t)=-N(t) \max _{s \in[0, T]} x(s), \quad t \in[0, T], \quad y(t)=0, \quad t \in[-h, 0]
$$

Thus, for $t \in[0, T]$, using the expression of $x(t)$ in Lemma 2.3, we obtain

$$
y(t)=-[\lambda A+M(t)]^{-1}\left[e^{-\hat{A}^{D} \hat{M} t} \int_{0}^{t} e^{\hat{A}^{D} \hat{M} \sigma} \hat{A}^{D} N(\sigma) \max _{s \in[0, T]} x(s) d \sigma+\left(I-\hat{A} \hat{A}^{D}\right) \hat{M}^{D} N(t) \max _{s \in[0, T]} x(s)\right] .
$$

Hence, we have

$$
\begin{aligned}
\max _{s \in[0, T]} x(s) \leqslant \max _{s \in[0, T]} y(s)= & \max _{s \in[0, T]}\left\{-[\lambda A+M(s)]^{-1}\left[e^{-\hat{A}^{D} \hat{M} s} \int_{0}^{s} e^{\hat{A}^{D} \hat{M} \sigma} \hat{A}^{D} N(\sigma) d \sigma\right.\right. \\
& \left.\left.+\left(I-\hat{A} \hat{A}^{D}\right) \hat{M}^{D} N(s)\right]\right\} \max _{s \in[0, T]} x(s)=M \max _{s \in[0, T]} x(s) .
\end{aligned}
$$

Using the condition $\left(\mathrm{H}_{2.4}\right)$, we have $\max _{s \in[0, T]} x(s) \leqslant 0$. Then, we obtain $x(t) \leqslant 0$ on $[0, T]$. Therefore, we conclude that $x(t) \leqslant 0$ on $[-h, T]$. The proof is complete.

Now, we will prove an existence result, which is necessary for our main results.

Lemma 2.5. Assume that the conditions $\left(\mathrm{H}_{2.1}\right)-\left(\mathrm{H}_{2.4}\right)$ hold, and

$\left(\mathrm{H}_{2.5}\right)$ the functions $\alpha_{0}, \beta_{0} \in \mathrm{C}^{0}\left([-\mathrm{h}, \mathrm{T}], \mathrm{R}^{\mathrm{n}}\right) \cup \mathrm{C}^{1}\left([0, \mathrm{~T}], \mathrm{R}^{\mathrm{n}}\right)$ are lower and upper solutions of IVP $(2.1)$, respectively, and $\alpha_{0}(\mathrm{t}) \leqslant \beta_{0}(\mathrm{t})$ on $[-\mathrm{h}, \mathrm{T}]$;

$\left(\mathrm{H}_{2.6}\right)$ the function $\mathrm{f} \in \mathrm{C}\left(\Omega\left(\alpha_{0}, \beta_{0}\right), \mathrm{R}^{\mathrm{n}}\right)$ satisfies the inequality

$$
f(t, y, v)-f(t, x, u) \leqslant-M(y-x)-N(v-u),
$$

where $y \leqslant x, v \leqslant u, M\left(t_{0}\right)=M, N\left(t_{0}\right)=N, t_{0} \in[0, T]$.

Then there exists a solution $x(t)$ of IVP (2.1) which satisfies $\alpha_{0}(t) \leqslant x(t) \leqslant \beta_{0}(t)$ on $[-h, T]$.

Proof. Consider the following iterative scheme

$$
\left\{\begin{array}{l}
A U_{n+1}^{\prime}(t)=Q_{n+1}(t)-M U_{n+1}(t)-N \max _{s \in[t-h, t]} U_{n+1}(s), t \in[0, T], \\
u_{n+1}(t)=\varphi(t), t \in[-h, 0],
\end{array}\right.
$$

where $Q_{n+1}(t)=f\left(t, U_{n}(t), \max _{s \in[t-h, t]} U_{n}(s)\right)+M U_{n}(t)+N \max _{s \in[t-h, t]} U_{n}(s)$. According to the iterative scheme, the sequences $\left\{\alpha_{\mathfrak{n}}(t)\right\}$ and $\left\{\beta_{\mathfrak{n}}(t)\right\}$ were generated by $\alpha_{0}(t)$ and $\beta_{0}(t)$, respectively.

Now, we will prove that

$$
\alpha_{0}(t) \leqslant \alpha_{1}(t) \leqslant \beta_{1}(t) \leqslant \beta_{0}(t), \quad t \in[-h, T] .
$$

For this purpose, setting $p_{1}(t)=\alpha_{0}(t)-\alpha_{1}(t)$, using the condition $\left(\mathrm{H}_{2.5}\right)$, we have

$$
A p_{1}^{\prime}(t) \leqslant-M p_{1}(t)-N \max _{s \in[t-h, t]} p_{1}(s), \quad t \in[0, T], \quad p_{1}(t) \leqslant 0, \quad t \in[-h, 0] .
$$

According to Lemma 2.4 , we have $p_{1}(t) \leqslant 0$ on $[-h, T]$. Similarly, setting $p_{2}(t)=\beta_{1}(t)-\beta_{0}(t)$, we can obtain $p_{2}(t) \leqslant 0$ on $[-h, T]$.

Letting $p_{3}(t)=\alpha_{1}(t)-\beta_{1}(t)$, from the condition $\left(H_{2.6}\right)$, we have

$$
A p_{3}^{\prime}(t) \leqslant-M p_{3}(t)-N \max _{s \in[t-h, t]} p_{3}(s), \quad t \in[0, T], \quad p_{3}(t)=0, \quad t \in[-h, 0] .
$$

By Lemma 2.4, we have $p_{3}(t) \leqslant 0$ on $[-h, T]$. 
The process can be continued to obtain that

$$
\alpha_{0}(t) \leqslant \alpha_{1}(t) \leqslant \cdots \leqslant \alpha_{n}(t) \leqslant \beta_{n}(t) \leqslant \cdots \leqslant \beta_{1}(t) \leqslant \beta_{0}(t), \quad t \in[-h, T] .
$$

It is easy to see that the sequence $\left\{\alpha_{n}(t)\right\}$ is uniformly bounded and equicontinuous, employing the Ascoli-Arzelà Theorem, the nondecreasing sequence $\left\{\alpha_{n}(t)\right\}$ converges pointwise to a function $x(t)$ that satisfies $\alpha_{0}(t) \leqslant x(t) \leqslant \beta_{0}(t)$. In view of IVP (2.5) and the Dominated Convergence Theorem, we obtain that $x(t)$ is a solution of

$$
A x^{\prime}(t)=f\left(t, x(t), \max _{s \in[t-h, t]} x(s)\right)-M(x(t)-x(t))-N(x(t)-x(t)), \quad t \in[0, T], \quad x(t)=\varphi(t), \quad t \in[-h, 0],
$$

that is, $x(t)$ is a solution of IVP (2.1). Therefore, we conclude that there exists a solution $x(t)$ of IVP (2.1) which satisfies $\alpha_{0}(t) \leqslant x(t) \leqslant \beta_{0}(t)$ on $[-h, T]$. The proof is complete.

\section{Main results}

In this section, we prove that the convergence of the sequence of successive approximations is of order $k$. Throughout this paper, we define $x^{i}=\left(x_{1}^{i}, x_{2}^{i}, \cdots, x_{n}^{i}\right)^{\top}, x^{i} y^{j}=\left(x_{1}^{i} y_{1}^{j}, x_{2}^{i} y_{2}^{j}, \cdots, x_{n}^{i} y_{n}^{j}\right)^{\top}$ for any $x, y \in R^{n}, i, j=1,2, \cdots$.

Theorem 3.1. Assume that the following conditions hold.

$\left(A_{3.1}\right)$ There exists a function $f \in C^{0,2 k+1,2 k+1}\left(\Omega\left(\alpha_{0}, \beta_{0}\right), R^{n}\right)$ such that the Fréchet derivatives $f_{y}(t, x, y) \geqslant 0$, $\frac{\partial^{2 k+1} f(t, x, y)}{\partial x^{i} y^{2 k+1-i}} \geqslant 0$ for $k \geqslant 1, i=0,1,2, \cdots, 2 k+1$.

$\left(\mathrm{A}_{3.2}\right)$ Conditions $\left(\mathrm{H}_{2.1}\right)-\left(\mathrm{H}_{2.4}\right)$ hold for $\mathrm{M}(\mathrm{t})=-\mathrm{f}_{\mathrm{x}}(\mathrm{t}, \mathrm{x}, \mathrm{y}), \mathrm{N}(\mathrm{t})=-\mathrm{f}_{\mathrm{y}}(\mathrm{t}, \mathrm{x}, \mathrm{y})$ with $(\mathrm{t}, \mathrm{x}, \mathrm{y}) \in \Omega\left(\alpha_{0}, \beta_{0}\right)$, and $\left(\mathrm{H}_{2.5}\right),\left(\mathrm{H}_{2.6}\right)$ hold.

Then there exist two sequences $\left\{\alpha_{n}(t)\right\}$ and $\left\{\beta_{n}(t)\right\}$ converging uniformly to the solution $x(t)$ of IVP (2.1) and the convergence is of order $2 \mathrm{k}+1$, that is, there exist positive matrices $\mathrm{K}_{1}, \mathrm{~K}_{2}$ such that for the solution $x(\mathrm{t})$ of IVP (2.1) in $\mathrm{S}\left(\alpha_{0}, \beta_{0}\right)$, the inequalities

$$
\left|x-\alpha_{n+1}\right|_{0} \leqslant K_{1}\left|x-\alpha_{n}\right|_{0}^{2 k+1}, \text { and }\left|\beta_{n+1}-x\right|_{0} \leqslant K_{2}\left|\beta_{n}-x\right|_{0}^{2 k+1}
$$

hold, where $|u|_{0}=\max _{s \in[-h, T]}|u(s)|=\left(\max _{s \in[-h, T]}\left|u_{1}(s)\right|, \max _{s \in[-h, T]}\left|u_{2}(s)\right|, \cdots, \max _{s \in[-h, T]}\left|u_{n}(s)\right|\right)^{\top}$.

Proof. From the condition $\left(\mathrm{A}_{3.1}\right)$, we have that

$$
\begin{aligned}
f\left(t, x(t), \max _{s \in[t-h, t]} x(s)\right) \geqslant & f\left(t, y(t), \max _{s \in[t-h, t]} y(s)\right)+\sum_{i=1}^{2 k} \frac{1}{i !}\left[(x(t)-y(t)) \frac{\partial}{\partial x}\right. \\
& \left.+\left(\max _{s \in[t-h, t]} x(s)-\max _{s \in[t-h, t]} y(s)\right) \frac{\partial}{\partial y}\right]^{i} f\left(t, y(t), \max _{s \in[t-h, t]} y(s)\right)
\end{aligned}
$$

for $\alpha_{0} \leqslant y \leqslant x \leqslant \beta_{0}$, and

$$
\begin{aligned}
f\left(t, x(t), \max _{s \in[t-h, t]} x(s)\right) \leqslant & f\left(t, y(t), \max _{s \in[t-h, t]} y(s)\right)+\sum_{i=1}^{2 k} \frac{1}{i !}\left[(x(t)-y(t)) \frac{\partial}{\partial x}\right. \\
& \left.+\left(\max _{s \in[t-h, t]} x(s)-\max _{s \in[t-h, t]} y(s)\right) \frac{\partial}{\partial y}\right]^{i} f\left(t, y(t), \max _{s \in[t-h, t]} y(s)\right)
\end{aligned}
$$

for $\alpha_{0} \leqslant x \leqslant y \leqslant \beta_{0}$, where

$$
\left[(x(t)-y(t)) \frac{\partial}{\partial x}+\left(\max _{s \in[t-h, t]} x(s)-\max _{s \in[t-h, t]} y(s)\right) \frac{\partial}{\partial y}\right]^{i} f\left(t, y(t), \max _{s \in[t-h, t]} y(s)\right)
$$




$$
=\sum_{k=0}^{i} C_{i}^{k} \frac{\partial^{i} f\left(t, y(t), \max _{s \in[t-h, t]} y(s)\right)}{\partial x^{k} \partial y^{i-k}}(x(t)-y(t))^{k}\left(\max _{s \in[t-h, t]} x(s)-\max _{s \in[t-h, t]} y(s)\right)^{i-k} .
$$

Let $\mathrm{L}_{\mathrm{m}}=\min _{s \in[-\mathrm{h}, 0]}\left(\varphi(s)-\alpha_{\mathrm{m}}(\mathrm{s})\right) \geqslant 0, \mathrm{C}_{\mathrm{m}}=\min _{\mathrm{s} \in[-\mathrm{h}, 0]}\left(\beta_{\mathrm{m}}(\mathrm{s})-\varphi(s)\right) \geqslant 0, \mathrm{k}_{\mathrm{m}}=\left(\mathrm{k}_{\mathrm{m} 1}, \mathrm{k}_{\mathrm{m} 2}, \cdots, \mathrm{k}_{\mathrm{mn}}\right)^{\top}$ and $p_{m}=\left(p_{m 1}, p_{m 2}, \cdots, p_{m n}\right)^{\top}$. Choose numbers $k_{m i}, p_{m i} \in[0,1)$ such that $k_{m i} \leqslant L_{m i}$ and $p_{m i} \leqslant C_{m i}$ for $i=1,2, \cdots, n ; m=0,1, \cdots, n, \cdots$.

Consider the following singular differential systems with "maxima"

$$
\left\{\begin{aligned}
A x^{\prime}(t)= & f\left(t, \alpha_{0}(t), \max _{s \in[t-h, t]} \alpha_{0}(s)\right)+\sum_{i=1}^{2 k} \frac{1}{i !}\left[\left(x(t)-\alpha_{0}(t)\right) \frac{\partial}{\partial x}\right. \\
& \left.+\left(\max _{s \in[t-h, t]} x(s)-\max _{s \in[t-h, t]} \alpha_{0}(s)\right) \frac{\partial}{\partial y}\right]^{i} f\left(t, \alpha_{0}(t), \max _{s \in[t-h, t]} \alpha_{0}(s)\right) \\
\equiv & F_{0}\left(t, x(t), \max _{s \in[t-h, t]} x(s)\right), \quad t \in[0, T] \\
x(t)= & \varphi(t)-k_{0}^{2 k} L_{0}, \quad t \in[-h, 0]
\end{aligned}\right.
$$

and

$$
\left\{\begin{aligned}
A x^{\prime}(t)= & f\left(t, \beta_{0}(t), \max _{s \in[t-h, t]} \beta_{0}(s)\right)+\sum_{i=1}^{2 k} \frac{1}{i !}\left[\left(x(t)-\beta_{0}(t)\right) \frac{\partial}{\partial x}\right. \\
& \left.+\left(\max _{s \in[t-h, t]} x(s)-\max _{s \in[t-h, t]} \beta_{0}(s)\right) \frac{\partial}{\partial y}\right]^{i} f\left(t, \beta_{0}(t), \max _{s \in[t-h, t]} \beta_{0}(s)\right) \\
\equiv & G_{0}\left(t, x(t), \max _{s \in[t-h, t]} x(s)\right), \quad t \in[0, T] \\
x(t)= & \varphi(t)+p_{0}^{2 k} C_{0}, t \in[-h, 0] .
\end{aligned}\right.
$$

Now, we prove that $\alpha_{0}(t)$ and $\beta_{0}(t)$ are lower and upper solutions of IVP (3.3), respectively. In fact, from the condition $\left(\mathrm{H}_{2.5}\right)$ and the choice of $\mathrm{k}_{0}, \mathrm{~L}_{0}$, we have

$$
\begin{aligned}
A & \alpha_{0}^{\prime}(t) \leqslant f\left(t, \alpha_{0}(t), \max _{s \in[t-h, t]} \alpha_{0}(s)\right)=F_{0}\left(t, \alpha_{0}(t), \max _{s \in[t-h, t]} \alpha_{0}(s)\right), \quad t \in[0, T], \\
\alpha_{0}(t) & \leqslant \varphi(t)-k_{0}^{2 k} L_{0}, \quad t \in[-h, 0],
\end{aligned}
$$

and by using the inequality (3.1), it follows that

$$
\begin{aligned}
A \beta_{0}^{\prime}(t) \geqslant & f\left(t, \alpha_{0}(t), \max _{s \in[t-h, t]} \alpha_{0}(s)\right)+\sum_{i=1}^{2 k} \frac{1}{i !}\left[\left(\beta_{0}(t)-\alpha_{0}(t)\right) \frac{\partial}{\partial x}\right. \\
& \left.+\left(\max _{s \in[t-h, t]} \beta_{0}(s)-\max _{s \in[t-h, t]} \alpha_{0}(s)\right) \frac{\partial}{\partial y}\right]^{i} f\left(t, \alpha_{0}(t), \max _{s \in[t-h, t]} \alpha_{0}(s)\right) \\
= & F_{0}\left(t, \beta_{0}(t), \max _{s \in[t-h, t]} \beta_{0}(s)\right), \quad t \in[0, T] \\
\beta_{0}(t) \geqslant & \varphi(t)-k_{0}^{2 k} L_{0}, \quad t \in[-h, 0] .
\end{aligned}
$$

Thus, $\alpha_{0}(t)$ and $\beta_{0}(t)$ are lower and upper solutions of IVP (3.3), respectively. Consequently, by Lemma 2.5 , there exists a solution $\alpha_{1}(t)$ of IVP (3.3) with $\alpha_{1}(t)=\varphi(t)-k_{0}^{2 k} L_{0}$ on $[-h, 0]$ such that $\alpha_{0}(t) \leqslant \alpha_{1}(t) \leqslant$ $\beta_{0}(t)$ on $[-h, T]$.

Similarly, applying the fact that $\alpha_{1}(t)$ is a solution of IVP (3.3), the inequalities (3.1), (3.2), the condition $\left(\mathrm{H}_{2.5}\right)$, and the choice of $\mathrm{p}_{0}, \mathrm{C}_{0}$, we obtain

$$
A \alpha_{1}^{\prime}(t)=f\left(t, \alpha_{0}(t), \max _{s \in[t-h, t]} \alpha_{0}(s)\right)+\sum_{i=1}^{2 k} \frac{1}{i !}\left[\left(\alpha_{1}(t)-\alpha_{0}(t)\right) \frac{\partial}{\partial x}\right.
$$




$$
\begin{aligned}
& \left.+\left(\max _{s \in[t-h, t]} \alpha_{1}(s)-\max _{s \in[t-h, t]} \alpha_{0}(s)\right) \frac{\partial}{\partial y}\right]^{i} f\left(t, \alpha_{0}(t), \max _{s \in[t-h, t]} \alpha_{0}(s)\right) \\
\leqslant & f\left(t, \alpha_{1}(t), \max _{s \in[t-h, t]} \alpha_{1}(s)\right) \\
\leqslant & f\left(t, \beta_{0}(t), \max _{s \in[t-h, t]} \beta_{0}(s)\right)+\sum_{i=1}^{2 k} \frac{1}{i !}\left[\left(\alpha_{1}(t)-\beta_{0}(t)\right) \frac{\partial}{\partial x}\right. \\
& \left.+\left(\max _{s \in[t-h, t]} \alpha_{1}(s)-\max _{s \in[t-h, t]} \beta_{0}(s)\right) \frac{\partial}{\partial y}\right]^{i} f\left(t, \beta_{0}(t), \max _{s \in[t-h, t]} \beta_{0}(s)\right) \\
\equiv & G_{0}\left(t, \alpha_{1}(t), \max _{s \in[t-h, t]} \alpha_{1}(s)\right), t \in[0, T], \\
\alpha_{1}(t) \leqslant & \varphi(t)+p_{0}^{2 k} C_{0}, t \in[-h, 0],
\end{aligned}
$$

and

$$
\begin{aligned}
& A \beta_{0}^{\prime}(t) \geqslant f\left(t, \beta_{0}(t), \max _{s \in[t-h, t]} \beta_{0}(s)\right)=G_{0}\left(t, \beta_{0}(t), \max _{s \in[t-h, t]} \beta_{0}(s)\right), t \in[0, T], \\
& \beta_{0}(t) \geqslant \varphi(t)+p_{0}^{2 k} C_{0}, t \in[-h, 0] .
\end{aligned}
$$

Thus, $\alpha_{1}(t)$ and $\beta_{0}(t)$ are lower and upper solutions of IVP (3.4), respectively. In view of Lemma 2.5, we see that there exists a solution $\beta_{1}(t)$ of IVP (3.4) with $\beta_{1}(t)=\varphi(t)+p_{0}^{2 k} C_{0}$ on $[-h, 0]$ such that $\alpha_{1}(t) \leqslant \beta_{1}(t) \leqslant \beta_{0}(t)$ on $[-h, T]$.

Next, we must show that $\alpha_{1}(t)$ and $\beta_{1}(t)$ are lower and upper solutions of IVP (2.1). For this purpose, using the conclusion that $\alpha_{1}(t)$ is a solution of IVP (3.3) and the inequality (3.1), we have

$$
\begin{aligned}
A \alpha_{1}^{\prime}(t)= & f\left(t, \alpha_{0}(t), \max _{s \in[t-h, t]} \alpha_{0}(s)\right)+\sum_{i=1}^{2 k} \frac{1}{i !}\left[\left(\alpha_{1}(t)-\alpha_{0}(t)\right) \frac{\partial}{\partial x}\right. \\
& \left.+\left(\max _{s \in[t-h, t]} \alpha_{1}(s)-\max _{s \in[t-h, t]} \alpha_{0}(s)\right) \frac{\partial}{\partial y}\right]^{i} f\left(t, \alpha_{0}(t), \max _{s \in[t-h, t]} \alpha_{0}(s)\right) \\
\leqslant & f\left(t, \alpha_{1}(t), \max _{s \in[t-h, t]} \alpha_{1}(s)\right), \quad t \in[0, T], \\
\alpha_{1}(t) \leqslant & \varphi(t), \quad t \in[-h, 0] .
\end{aligned}
$$

This implies that $\alpha_{1}(t)$ is a lower solution of IVP (2.1) on $[-h, T]$. Similar arguments show that

$$
A \beta_{1}^{\prime}(t) \geqslant f\left(t, \beta_{1}(t), \max _{s \in[t-h, t]} \beta_{1}(s)\right), \quad t \in[0, T], \quad \beta_{1}(t) \geqslant \varphi(t), t \in[-h, 0] .
$$

Thus, $\beta_{1}(t)$ is an upper solution of IVP $(2.1)$ on $[-h, T]$. Then, we obtain

$$
\alpha_{0}(t) \leqslant \alpha_{1}(t) \leqslant \beta_{1}(t) \leqslant \beta_{0}(t), \quad t \in[-h, T] .
$$

Now, we assume that $\alpha_{n}(t)$ and $\beta_{n}(t)$ are lower and upper solutions of IVP (2.1), respectively, and $\alpha_{n}(t) \leqslant \beta_{n}(t)$ on $[-h, T]$. We need to show that

$$
\alpha_{\mathfrak{n}}(t) \leqslant \alpha_{n+1}(t) \leqslant \beta_{n+1}(t) \leqslant \beta_{n}(t), \quad t \in[-h, T],
$$

where $\alpha_{n+1}(t)$ and $\beta_{n+1}(t)$ are solutions of the following singular differential systems

$$
\left\{\begin{aligned}
A x^{\prime}(t)= & f\left(t, \alpha_{n}(t), \max _{s \in[t-h, t]} \alpha_{n}(s)\right)+\sum_{i=1}^{2 k} \frac{1}{i !}\left[\left(x(t)-\alpha_{n}(t)\right) \frac{\partial}{\partial x}\right. \\
& \left.+\left(\max _{s \in[t-h, t]} x(s)-\max _{s \in[t-h, t]} \alpha_{n}(s)\right) \frac{\partial}{\partial y}\right]^{i} f\left(t, \alpha_{n}(t), \max _{s \in[t-h, t]} \alpha_{n}(s)\right) \\
\equiv & F_{n}\left(t, x(t), \max _{s \in[t-h, t]} x(s)\right), \quad t \in[0, T], \\
x(t)= & \varphi(t)-k_{n}^{2 k} L_{n}, t \in[-h, 0],
\end{aligned}\right.
$$


and

$$
\left\{\begin{aligned}
A x^{\prime}(t)= & f\left(t, \beta_{n}(t), \max _{s \in[t-h, t]} \beta_{n}(s)\right)+\sum_{i=1}^{2 k} \frac{1}{i !}\left[\left(x(t)-\beta_{n}(t)\right) \frac{\partial}{\partial x}\right. \\
& \left.+\left(\max _{s \in[t-h, t]} x(s)-\max _{s \in[t-h, t]} \beta_{n}(s)\right) \frac{\partial}{\partial y}\right]^{i} f\left(t, \beta_{n}(t), \max _{s \in[t-h, t]} \beta_{n}(s)\right) \\
\equiv & G_{n}\left(t, x(t), \max _{s \in[t-h, t]} x(s)\right), \quad t \in[0, T], \\
x(t)= & \varphi(t)+p_{n}^{2 k} C_{n}, \quad t \in[-h, 0] .
\end{aligned}\right.
$$

From the assumption that $\alpha_{n}(t)$ is a lower solution of IVP (2.1), we get that

$$
\begin{aligned}
A \alpha_{n}^{\prime}(t) & \leqslant f\left(t, \alpha_{n}(t), \max _{s \in[t-h, t]} \alpha_{n}(s)\right)=F_{n}\left(t, \alpha_{n}(t), \max _{s \in[t-h, t]} \alpha_{n}(s)\right), t \in[0, T], \\
& \alpha_{n}(t) \leqslant \varphi(t)-k_{n}^{2 k} L_{n}, \quad t \in[-h, 0] .
\end{aligned}
$$

The assumption that $\beta_{n}(t)$ is an upper solution of IVP (2.1), together with the inequality (3.1), implies

$$
\begin{aligned}
A \beta_{n}^{\prime}(t) \geqslant & f\left(t, \alpha_{n}(t), \max _{s \in[t-h, t]} \alpha_{n}(s)\right)+\sum_{i=1}^{2 k} \frac{1}{i !}\left[\left(\beta_{n}(t)-\alpha_{n}(t)\right) \frac{\partial}{\partial x}\right. \\
& \left.+\left(\max _{s \in[t-h, t]} \beta_{n}(s)-\max _{s \in[t-h, t]} \alpha_{n}(s)\right) \frac{\partial}{\partial y}\right]^{i} f\left(t, \alpha_{n}(t), \max _{s \in[t-h, t]} \alpha_{n}(s)\right) \\
= & F_{n}\left(t, \beta_{n}(t), \max _{s \in[t-h, t]} \beta_{n}(s)\right), \quad t \in[0, T], \\
\beta_{n}(t) \geqslant & \varphi(t)-k_{n}^{2 k} L_{n}, t \in[-h, 0] .
\end{aligned}
$$

Thus, $\alpha_{n}(t)$ and $\beta_{n}(t)$ are lower and upper solutions of IVP (3.5), respectively. According to Lemma 2.5, we conclude that the IVP (3.5) has a solution $\alpha_{n+1}(t)$ with $\alpha_{n+1}(t)=\varphi(t)-k_{n}^{2 k} L_{n}$ on $[-h, 0]$ such that $\alpha_{n}(t) \leqslant \alpha_{n+1}(t) \leqslant \beta_{n}(t)$ on $[-h, T]$. Similarly, we can show that IVP (3.6) has a solution $\beta_{n+1}(t)$ such that $\alpha_{n+1}(t) \leqslant \beta_{n+1}(t) \leqslant \beta_{n}(t)$ on $[-h, T]$.

Now, we come to prove that $\alpha_{n+1}(t)$ and $\beta_{n+1}(t)$ are lower and upper solutions of IVP (2.1). Using the conclusion that $\alpha_{n+1}(t)$ is a solution of IVP (3.5) and the inequality (3.1), we obtain

$$
\begin{aligned}
A \alpha_{n+1}^{\prime}(t)= & f\left(t, \alpha_{n}(t), \max _{s \in[t-h, t]} \alpha_{n}(s)\right)+\sum_{i=1}^{2 k} \frac{1}{i !}\left[\left(\alpha_{n+1}(t)-\alpha_{n}(t)\right) \frac{\partial}{\partial x}\right. \\
& \left.+\left(\max _{s \in[t-h, t]} \alpha_{n+1}(s)-\max _{s \in[t-h, t]} \alpha_{n}(s)\right) \frac{\partial}{\partial y}\right]^{i} f\left(t, \alpha_{n}(t), \max _{s \in[t-h, t]} \alpha_{n}(s)\right) \\
\leqslant & f\left(t, \alpha_{n+1}(t), \max _{s \in[t-h, t]} \alpha_{n+1}(s)\right), \quad t \in[0, T], \\
\alpha_{n+1}(t) \leqslant & \varphi(t), t \in[-h, 0] .
\end{aligned}
$$

Analogously, we can prove that $\beta_{n+1}(t)$ is an upper solution of IVP (2.1). Then, we conclude that

$$
\alpha_{n}(t) \leqslant \alpha_{n+1}(t) \leqslant \beta_{n+1}(t) \leqslant \beta_{n}(t), \quad t \in[-h, T] .
$$

By induction, we have

$$
\alpha_{0}(t) \leqslant \alpha_{1}(t) \leqslant \cdots \leqslant \alpha_{n}(t) \leqslant \beta_{n}(t) \leqslant \cdots \leqslant \beta_{1}(t) \leqslant \beta_{0}(t), \quad t \in[-h, T] .
$$

We can show easily that the sequences $\left\{\alpha_{n}(t)\right\}$ and $\left\{\beta_{n}(t)\right\}$ are uniformly bounded and equicontinuous. Hence, employing Ascoli-Arzelà Theorem, we have that both monotone sequences $\left\{\alpha_{n}(t)\right\}$ and $\left\{\beta_{n}(t)\right\}$ have pointwise limits on $[-h, T]$. Taking the limit as $n \rightarrow \infty$, we get that

$$
\lim _{n \rightarrow \infty} \alpha_{n}(t)=\rho(t) \leqslant r(t)=\lim _{n \rightarrow \infty} \beta_{n}(t) .
$$


Next, we show that $\rho(t) \geqslant r(t)$ on $[-h, T]$. From the IVPs (3.5) and (3.6), we can show easily that $\rho(t)$ and $r(t)$ are solutions of IVP (2.1). For $t \in[-h, 0]$, it is clear that $\rho(t)=r(t)$. For $t \in[0, T]$, the condition $\left(\mathrm{A}_{3.1}\right)$ yields

$$
\begin{aligned}
A r^{\prime}(t)-A \rho^{\prime}(t)= & f\left(t, r(t), \max _{s \in[t-h, t]} r(s)\right)-f\left(t, \rho(t), \max _{s \in[t-h, t]} \rho(s)\right) \\
= & \left(\int_{0}^{1} f_{x}\left(t, \sigma r(t)+(1-\sigma) \rho(t), \max _{s \in[t-h, t]} r(s)\right) d \sigma\right)(r(t)-\rho(t)) \\
& +\left(\int_{0}^{1} f_{y}\left(t, \rho(t), \sigma \max _{s \in[t-h, t]} r(s)+(1-\sigma) \max _{s \in[t-h, t]} \rho(s)\right) d \sigma\right) \\
& \times\left(\max _{s \in[t-h, t]} r(s)-\max _{s \in[t-h, t]} \rho(s)\right) \\
\leqslant & \left(\int_{0}^{1} f_{x}\left(t, \sigma r(t)+(1-\sigma) \rho(t), \max _{s \in[t-h, t]} r(s)\right) d \sigma\right)(r(t)-\rho(t)) \\
& +\left(\int_{0}^{1} f_{y}\left(t, \rho(t), \sigma \max _{s \in[t-h, t]} r(s)+(1-\sigma) \max _{s \in[t-h, t]} \rho(s)\right) d \sigma\right) \times \max _{s \in[t-h, t]}(r(s)-\rho(s)) \\
= & -M(t)(r(t)-\rho(t))-N(t) \max _{s \in[t-h, t]}(r(s)-\rho(s)) .
\end{aligned}
$$

According to Lemma 2.4, we get $r(t) \leqslant \rho(t)$ on $[0, T]$. Then, we conclude that $\rho(t) \equiv r(t)$ on $[-h, T]$. Hence, $\left\{\alpha_{n}(t)\right\}$ and $\left\{\beta_{n}(t)\right\}$ converge to the unique solution of IVP (2.1) when $f_{x}$ and $f_{y}$ exist and are continuous.

Finally, we shall show that the convergence of the sequences $\left\{\alpha_{n}(t)\right\}$ and $\left\{\beta_{n}(t)\right\}$ to the solution $x(t)$ of IVP (2.1) is of order $2 k+1$. For this purpose, consider

$$
a_{n+1}(t)=x(t)-\alpha_{n+1}(t) \geqslant 0, \quad t \in[-h, T] .
$$

Case 1. If $t \in[-h, 0]$, since $x(t)-\alpha_{n+1}(t)=k_{n}^{2 k} L_{n}$ and $k_{n i} \leqslant L_{n i}$, we have

$$
x(t)-\alpha_{n+1}(t)=k_{n}^{2 k} L_{n} \leqslant\left(L_{n}\right)^{2 k+1}=\left(\min _{s \in[-h, 0]}\left(x(s)-\alpha_{n}(s)\right)\right)^{2 k+1} \leqslant \max _{s \in[-h, 0]}\left|x(s)-\alpha_{n}(s)\right|^{2 k+1},
$$

which implies that

$$
a_{n+1}(t) \leqslant \max _{s \in[-h, 0]}\left|a_{n}(s)\right|^{2 k+1} .
$$

Case 2. If $t \in[0, T]$, from the condition $\left(\mathrm{A}_{3.1}\right)$ and the mean value theorem, we obtain

$$
\begin{aligned}
A a_{n+1}^{\prime}(t)= & f\left(t, x(t), \max _{s \in[t-h, t]} x(s)\right)-\left\{f\left(t, \alpha_{n}(t), \max _{s \in[t-h, t]} \alpha_{n}(s)\right)+\sum_{i=1}^{2 k} \frac{1}{i !}\left[\left(\alpha_{n+1}(t)-\alpha_{n}(t)\right) \frac{\partial}{\partial x}\right.\right. \\
& \left.\left.+\left(\max _{s \in[t-h, t]} \alpha_{n+1}(s)-\max _{s \in[t-h, t]} \alpha_{n}(s)\right) \frac{\partial}{\partial y}\right]^{i} f\left(t, \alpha_{n}(t), \max _{s \in[t-h, t]} \alpha_{n}(s)\right)\right\} \\
= & f\left(t, x(t), \max _{s \in[t-h, t]} x(s)\right)-\left\{f\left(t, \alpha_{n+1}(t), \max _{s \in[t-h, t]} \alpha_{n+1}(s)\right)\right. \\
& -\frac{1}{2 k+1 !} \int_{0}^{1}\left[\left(\alpha_{n+1}(t)-\alpha_{n}(t)\right) \frac{\partial}{\partial x}+\left(\max _{s \in[t-h, t]} \alpha_{n+1}(s)-\max _{s \in[t-h, t]} \alpha_{n}(s)\right) \frac{\partial}{\partial y}\right]^{2 k+1} \\
& \left.\times f\left(t, \sigma \alpha_{n+1}(t)+(1-\sigma) \alpha_{n}(t), \sigma \max _{s \in[t-h, t]} \alpha_{n+1}(s)+(1-\sigma) \max _{s \in[t-h, t]} \alpha_{n}(s)\right) d \sigma\right\} \\
\leqslant & \left(\int_{0}^{1} f_{x}\left(t, \sigma x(t)+(1-\sigma) \alpha_{n+1}(t), \max _{s \in[t-h, t]} x(s)\right) d \sigma\right)\left(x(t)-\alpha_{n+1}(t)\right)
\end{aligned}
$$




$$
\begin{aligned}
& +\left(\int_{0}^{1} f_{y}\left(t, \alpha_{n+1}(t), \sigma \max _{s \in[t-h, t]} x(s)+(1-\sigma) \max _{s \in[t-h, t]} \alpha_{n+1}(s)\right) d \sigma\right) \\
& \times \max _{s \in[t-h, t]}\left(x(s)-\alpha_{n+1}(s)\right)+2^{2 k+1} M_{1} \max _{s \in[t-h, t]}\left|a_{n}(s)\right|^{2 k+1} \\
= & -M(t) a_{n+1}(t)-N(t) \max _{s \in[t-h, t]} a_{n+1}(s)+2^{2 k+1} M_{1} \max _{s \in[t-h, t]}\left|a_{n}(s)\right|^{2 k+1} \\
\leqslant & -M(t) a_{n+1}(t)-N(t) \max _{s \in[-h, T]} a_{n+1}(s)+2^{2 k+1} M_{1} \max _{s \in[-h, T]}\left|a_{n}(s)\right|^{2 k+1},
\end{aligned}
$$

where $\frac{1}{(2 k+1) !} \int_{0}^{1}\left[(x-y) \frac{\partial}{\partial x}+\left(\max _{s \in[t-h, t]} x(s)-\max _{s \in[t-h, t]} y(s)\right) \frac{\partial}{\partial y}\right]^{2 k+1} f\left(t, \sigma x+(1-\sigma) y, \sigma \max _{s \in[t-h, t]} x(s)+(1-\right.$ б) $\left.\max _{s \in[t-h, t]} y(s)\right) d \sigma \leqslant 2^{2 k+1} M_{1}\left(\max _{s \in[t-h, t]}(x(s)-y(s))\right)^{2 k+1}$ for $\alpha_{0} \leqslant y \leqslant x \leqslant \beta_{0}$, and $M_{1}$ is a positive $n \times n$ matrix. According to Lemma 2.2, we have $a_{n+1}(t) \leqslant u(t)$ on $[0, T]$, where $u(t)$ is the solution of

$$
\begin{aligned}
A u^{\prime}(t)+M(t) u(t) & =-N(t) \max _{s \in[-h, T]} a_{n+1}(s)+2^{2 k+1} M_{1} \max _{s \in[-h, T]}\left|a_{n}(s)\right|^{2 k+1}, t \in[0, T], \\
u(t) & =k_{n}^{2 k} L_{n}, t \in[-h, 0] .
\end{aligned}
$$

Hence, using the expression of $x(t)$ in Lemma 2.3, we get

$$
\begin{aligned}
& \max _{s \in[-h, T]} a_{n+1}(s) \\
& \leqslant\left\{I-\max _{s \in[0, T]}\left\{-[\lambda A+M(s)]^{-1}\left[e^{-\hat{A}^{D} \hat{M} s} \int_{0}^{s} e^{\hat{A}^{D} \hat{M} \sigma} \hat{A}^{D} N(\sigma) d \sigma\right.\right.\right. \\
&\left.\left.\left.+\left(I-\hat{A} \hat{A}^{D}\right) \hat{M}^{D} N(s)\right]\right\}\right\}^{-1} \max _{s \in[0, T]}\left\{[ \lambda A + M ( s ) ] ^ { - 1 } \left[e^{-\hat{A}^{D} \hat{M} s} \hat{A} \hat{A}^{D}[\lambda A+M(s)] k_{n}^{2 k} L_{n}\right.\right. \\
&+e^{-\hat{A}^{D} \hat{M} s} \int_{0}^{s} e^{\hat{A}^{D} \hat{M} \sigma} \hat{A}^{D} 2^{2 k+1} M_{1} \max _{s \in[-h, T]}\left|a_{n}(s)\right|^{2 k+1} d \sigma \\
&\left.\left.+\left(I-\hat{A} \hat{A}^{D}\right) \hat{M}^{D} 2^{2 k+1} M_{1} \max _{s \in[-h, T]}\left|a_{n}(s)\right|^{2 k+1}\right]\right\} .
\end{aligned}
$$

Thus, by suitable estimates, we conclude from the inequalities (3.7) and (3.8) that the following inequality holds

$$
\left|a_{n+1}\right|_{0} \leqslant K_{1}\left|a_{n}\right|_{0}^{2 k+1},
$$

where $K_{1}$ is a positive matrix, and $|a|_{0}=\max _{s \in[-h, T]}|a(s)|=\left(\max _{s \in[-h, T]}\left|a_{1}(s)\right|, \cdots, \max _{s \in[-h, T]}\left|a_{n}(s)\right|\right)^{T}$. This proves that the convergence of the sequence $\left\{\alpha_{n}(t)\right\}$ is of order $2 k+1$.

Similarly, consider

$$
b_{n+1}(t)=\beta_{n+1}(t)-x(t) \geqslant 0, \quad t \in[-h, T] .
$$

Case 1. If $t \in[-h, 0]$, in view of the fact that $\beta_{n+1}(t)-x(t)=p_{n}^{2 k} C_{n}$ and $p_{n i} \leqslant C_{n i}$, we get

$$
\beta_{n+1}(t)-x(t)=p_{n}^{2 k} C_{n} \leqslant\left(C_{n}\right)^{2 k+1}=\left(\min _{s \in[-h, 0]}\left(\beta_{n}(s)-x(s)\right)\right)^{2 k+1} \leqslant \max _{s \in[-h, 0]}\left|\beta_{n}(s)-x(s)\right|^{2 k+1},
$$

which shows that

$$
b_{n+1}(t) \leqslant \max _{s \in[-h, 0]}\left|b_{n}(s)\right|^{2 k+1} .
$$

Case 2. If $t \in[0, T]$, utilizing the condition $\left(A_{3.1}\right)$ and the mean value theorem, we have

$$
A b_{n+1}^{\prime}(t)=f\left(t, \beta_{n}(t), \max _{s \in[t-h, t]} \beta_{n}(s)\right)+\sum_{i=1}^{2 k} \frac{1}{i !}\left[\left(\beta_{n+1}(t)-\beta_{\mathfrak{n}}(t)\right) \frac{\partial}{\partial x}\right.
$$




$$
\begin{aligned}
& \left.+\left(\max _{s \in[t-h, t]} \beta_{n+1}(s)-\max _{s \in[t-h, t]} \beta_{n}(s)\right) \frac{\partial}{\partial y}\right]^{i} f\left(t, \beta_{n}(t), \max _{s \in[t-h, t]} \beta_{n}(s)\right) \\
& -f\left(t, x(t), \max _{s \in[t-h, t]} x(s)\right) \\
= & f\left(t, \beta_{n+1}(t), \max _{s \in[t-h, t]} \beta_{n+1}(s)\right)-\frac{1}{(2 k+1) !} \int_{0}^{1}\left[\left(\beta_{n+1}(t)-\beta_{n}(t)\right) \frac{\partial}{\partial x}\right. \\
& \left.+\left(\max _{s \in[t-h, t]} \beta_{n+1}(s)-\max _{s \in[t-h, t]} \beta_{n}(s)\right) \frac{\partial}{\partial y}\right]^{2 k+1} \\
& \times f\left(t, \sigma \beta_{n+1}(t)+(1-\sigma) \beta_{n}(t), \sigma \max _{s \in[t-h, t]} \beta_{n+1}(s)+(1-\sigma) \max _{s \in[t-h, t]} \beta_{n}(s)\right) d \sigma \\
& -f\left(t, x(t), \max _{s \in[t-h, t]} x(s)\right) \\
\leqslant & \left(\int_{0}^{1} f_{x}\left(t, \sigma \beta_{n+1}(t)+(1-\sigma) x(t), \max _{s \in[t-h, t]} \beta_{n+1}(s)\right) d \sigma\right)\left(\beta_{n+1}(t)-x(t)\right) \\
& +\left(\int _ { 0 } ^ { 1 } f _ { y } \left(t, x(t), \sigma \max _{s \in[t-h, t]} \beta_{n+1}(s)+(1-\sigma)\right.\right. \\
& \times \max _{s \in[t-h, t]}\left(\beta_{n+1}(s)-x(s)\right)+2^{2 k+1} M_{1} \max _{s \in[t-h, t]}\left|b_{n}(s)\right|^{2 k+1} \\
= & -M(t)) d \sigma) \\
\leqslant & -M(t) b_{n+1}(t)-N(t) \max _{s \in[t-h, t]} b_{n+1}(s)+2^{2 k+1} M_{1} \max _{s \in[t-h, t]}\left|b_{n}(s)\right|^{2 k+1}
\end{aligned}
$$

By Lemma 2.2, we obtain $b_{n+1}(t) \leqslant u(t)$ on $[0, T]$, where $u(t)$ is the solution of

$$
\begin{aligned}
& A u^{\prime}(t)+M(t) u(t)=-N(t) \max _{s \in[-h, T]} b_{n+1}(s)+2^{2 k+1} M_{1} \max _{s \in[-h, T]}\left|b_{n}(s)\right|^{2 k+1}, t \in[0, T], \\
& u(t)=p_{n}^{2 k} C_{n}, t \in[-h, 0] .
\end{aligned}
$$

Hence, using the expression of $x(t)$ in Lemma 2.3, we get

$$
\begin{aligned}
& \max _{s \in[-h, T]} b_{n+1}(s) \\
& \leqslant\left\{I-\max _{s \in[0, T]}\left\{-[\lambda A+M(s)]^{-1}\left[e^{-\hat{A}^{D} \hat{M} s} \int_{0}^{s} e^{\hat{A}^{D} \hat{M} \sigma} \hat{A}^{D} N(\sigma) d \sigma\right.\right.\right. \\
&\left.\left.\left.+\left(I-\hat{A} \hat{A}^{D}\right) \hat{M}^{D} N(s)\right]\right\}\right\}^{-1} \max _{s \in[0, T]}\left\{[ \lambda A + M ( s ) ] ^ { - 1 } \left[e^{-\hat{A}^{D} \hat{M} s} \hat{A} \hat{A}^{D}[\lambda A+M(s)] k_{n}^{2 k} L_{n}\right.\right. \\
&+e^{-\hat{A}^{D} \hat{M} s} \int_{0}^{s} e^{\hat{A}^{D} \hat{M} \sigma} \hat{A}^{D} 2^{2 k+1} M_{1} \max _{s \in[-h, T]}\left|b_{n}(s)\right|^{2 k+1} d \sigma \\
&\left.\left.+\left(I-\hat{A} \hat{A}^{D}\right) \hat{M}^{D} 2^{2 k+1} M_{1} \max _{s \in[-h, T]}\left|b_{n}(s)\right|^{2 k+1}\right]\right\} .
\end{aligned}
$$

Consequently, from the inequalities (3.9) and (3.10), and making suitable estimates, we get

$$
\left|b_{n+1}\right|_{0} \leqslant K_{2}\left|b_{n}\right|_{0}^{2 k+1},
$$

where $K_{2}$ is a positive matrix. This shows that the convergence of the sequence $\left\{\beta_{n}(t)\right\}$ is of order $2 k+1$. The proof is complete.

Theorem 3.2. Assume that the condition $\left(\mathrm{A}_{3.2}\right)$ holds, and

$\left(\mathrm{A}_{3.3}\right)$ there exists a function $\mathrm{f} \in \mathrm{C}^{0,2 \mathrm{k}, 2 \mathrm{k}}\left(\Omega\left(\alpha_{0}, \beta_{0}\right), \mathrm{R}^{\mathrm{n}}\right)$ such that the Fréchet derivatives $\mathrm{f}_{\mathrm{y}}(\mathrm{t}, \mathrm{x}, \mathrm{y}) \geqslant 0$, $\frac{\partial^{2 k} f(t, x, y)}{\partial x^{i} y^{2 k-i}} \geqslant 0$ for $k \geqslant 1, i=0,1,2, \cdots, 2 k$. 
Then there exist two sequences $\left\{\alpha_{n}(t)\right\}$ and $\left\{\beta_{n}(t)\right\}$ converging uniformly to the solution $x(t)$ of IVP (2.1) and the convergence is of order $2 \mathrm{k}$, that is, there exist positive matrices $\mathrm{K}_{3}, \mathrm{~K}_{4}$ such that for the solution $\mathrm{x}(\mathrm{t})$ of IVP (2.1) in $S\left(\alpha_{0}, \beta_{0}\right)$, the inequalities

$$
\left|x-\alpha_{n+1}\right|_{0} \leqslant K_{3}\left|x-\alpha_{n}\right|_{0}^{2 k}, \quad\left|\beta_{n+1}-x\right|_{0} \leqslant K_{4}\left|\beta_{n}-x\right|_{0}^{2 k-1}\left(\left|\beta_{n}-x\right|_{0}+\left|x-\alpha_{n}\right|_{0}\right)
$$

hold, where $|\mathfrak{u}|_{0}=\max _{s \in[-h, T]}|\mathfrak{u}(s)|=\left(\max _{s \in[-h, T]}\left|\mathfrak{u}_{1}(s)\right|, \max _{s \in[-h, T]}\left|u_{2}(s)\right|, \cdots, \max _{s \in[-h, T]}\left|u_{n}(s)\right|\right)^{\top}$.

Proof. In view of the condition $\left(\mathrm{A}_{3.3}\right)$, we have

$$
\begin{aligned}
f\left(t, x(t), \max _{s \in[t-h, t]} x(s)\right) \geqslant & f\left(t, y(t), \max _{s \in[t-h, t]} y(s)\right)+\sum_{i=1}^{2 k-1} \frac{1}{i !}\left[(x(t)-y(t)) \frac{\partial}{\partial x}\right. \\
& \left.+\left(\max _{s \in[t-h, t]} x(s)-\max _{s \in[t-h, t]} y(s)\right) \frac{\partial}{\partial y}\right]^{i} f\left(t, y(t), \max _{s \in[t-h, t]} y(s)\right)
\end{aligned}
$$

for $\alpha_{0} \leqslant y \leqslant x \leqslant \beta_{0}$, and

$$
\begin{aligned}
f\left(t, x(t), \max _{s \in[t-h, t]} x(s)\right) \leqslant & f\left(t, y(t), \max _{s \in[t-h, t]} y(s)\right)+\sum_{i=1}^{2 k-2} \frac{1}{i !}\left[(x(t)-y(t)) \frac{\partial}{\partial x}\right. \\
& \left.+\left(\max _{s \in[t-h, t]} x(s)-\max _{s \in[t-h, t]} y(s)\right) \frac{\partial}{\partial y}\right]^{i} f\left(t, y(t), \max _{s \in[t-h, t]} y(s)\right) \\
& +\frac{1}{(2 k-1) !}\left[(x(t)-y(t)) \frac{\partial}{\partial x}\right. \\
& \left.+\left(\max _{s \in[t-h, t]} x(s)-\max _{s \in[t-h, t]} y(s)\right) \frac{\partial}{\partial y}\right]^{2 k-1} f\left(t, x(t), \max _{s \in[t-h, t]} x(s)\right)
\end{aligned}
$$

for $\alpha_{0} \leqslant x \leqslant y \leqslant \beta_{0}$.

We consider the singular differential systems with "maxima"

$$
\left\{\begin{aligned}
A x^{\prime}(t)= & f\left(t, \alpha_{0}(t), \max _{s \in[t-h, t]} \alpha_{0}(s)\right)+\sum_{i=1}^{2 k-1} \frac{1}{i !}\left[\left(x(t)-\alpha_{0}(t)\right) \frac{\partial}{\partial x}\right. \\
& \left.+\left(\max _{s \in[t-h, t]} x(s)-\max _{s \in[t-h, t]} \alpha_{0}(s)\right) \frac{\partial}{\partial y}\right]^{i} f\left(t, \alpha_{0}(t), \max _{s \in[t-h, t]} \alpha_{0}(s)\right) \\
\equiv & F_{0}\left(t, x(t), \max _{s \in[t-h, t]} x(s)\right), \quad t \in[0, T] \\
x(t)= & \varphi(t)-k_{0}^{2 k-1} L_{0}, \quad t \in[-h, 0]
\end{aligned}\right.
$$

and

$$
\left\{\begin{aligned}
A x^{\prime}(t)= & f\left(t, \beta_{0}(t), \max _{s \in[t-h, t]} \beta_{0}(s)\right)+\sum_{i=1}^{2 k-2} \frac{1}{i !}\left[\left(x(t)-\beta_{0}(t)\right) \frac{\partial}{\partial x}\right. \\
& \left.+\left(\max _{s \in[t-h, t]} x(s)-\max _{s \in[t-h, t]} \beta_{0}(s)\right) \frac{\partial}{\partial y}\right]^{i} f\left(t, \beta_{0}(t), \max _{s \in[t-h, t]} \beta_{0}(s)\right) \\
& +\frac{1}{(2 k-1) !}\left[\left(x(t)-\beta_{0}(t)\right) \frac{\partial}{\partial x}\right. \\
& \left.+\left(\max _{s \in[t-h, t]} x(s)-\max _{s \in[t-h, t]} \beta_{0}(s)\right) \frac{\partial}{\partial y}\right]^{2 k-1} f\left(t, \alpha_{0}(t), \max _{s \in[t-h, t]} \alpha_{0}(s)\right) \\
\equiv & G_{0}\left(t, x(t), \max _{s \in[t-h, t]} x(s)\right), t \in[0, T], \\
x(t)= & \varphi(t)+p_{0}^{2 k-1} C_{0}, t \in[-h, 0] .
\end{aligned}\right.
$$


Similar to the proof of Theorem 3.1, we can show that there exists a solution $\alpha_{1}(t)$ of IVP (3.13) with $\alpha_{1}(t)=\varphi(t)-k_{0}^{2 k-1} L_{0}$ on $[-h, 0]$ such that $\alpha_{0}(t) \leqslant \alpha_{1}(t) \leqslant \beta_{0}(t)$ on $[-h, T]$.

We shall now show that $\alpha_{1}(t)$ and $\beta_{0}(t)$ are lower and upper solutions of IVP (3.14), respectively. In view of the fact that $\alpha_{1}(t)$ is a solution of IVP (3.13), the conditions $\left(\mathrm{H}_{2.5}\right),\left(\mathrm{A}_{3.3}\right)$, the inequalities (3.11), (3.12), and the choice of $\mathrm{p}_{0}, \mathrm{C}_{0}$, we obtain

$$
\begin{aligned}
A \alpha_{1}^{\prime}(t) \leqslant & f\left(t, \beta_{0}(t), \max _{s \in[t-h, t]} \beta_{0}(s)\right)+\sum_{i=1}^{2 k-2} \frac{1}{i !}\left[\left(\alpha_{1}(t)-\beta_{0}(t)\right) \frac{\partial}{\partial x}\right. \\
& \left.+\left(\max _{s \in[t-h, t]} \alpha_{1}(s)-\max _{s \in[t-h, t]} \beta_{0}(s)\right) \frac{\partial}{\partial y}\right]^{i} f\left(t, \beta_{0}(t), \max _{s \in[t-h, t]} \beta_{0}(s)\right) \\
& +\frac{1}{(2 k-1) !}\left[\left(\alpha_{1}(t)-\beta_{0}(t)\right) \frac{\partial}{\partial x}\right. \\
& \left.+\left(\max _{s \in[t-h, t]} \alpha_{1}(s)-\max _{s \in[t-h, t]} \beta_{0}(s)\right) \frac{\partial}{\partial y}\right]^{2 k-1} f\left(t, \alpha_{1}(t), \max _{s \in[t-h, t]} \alpha_{1}(s)\right) \\
\leqslant & f\left(t, \beta_{0}(t), \max _{s \in[t-h, t]} \beta_{0}(s)\right)+\sum_{i=1}^{2 k-2} \frac{1}{i !}\left[\left(\alpha_{1}(t)-\beta_{0}(t)\right) \frac{\partial}{\partial x}\right. \\
& \left.+\left(\max _{s \in[t-h, t]} \alpha_{1}(s)-\max _{s \in[t-h, t]} \beta_{0}(s)\right) \frac{\partial}{\partial y}\right]^{i} f\left(t, \beta_{0}(t), \max _{s \in[t-h, t]} \beta_{0}(s)\right) \\
& +\frac{1}{(2 k-1) !}\left[\left(\alpha_{1}(t)-\beta_{0}(t)\right) \frac{\partial}{\partial x}\right. \\
& \left.+\left(\max _{s \in[t-h, t]} \alpha_{1}(s)-\max _{s \in[t-h, t]} \beta_{0}(s)\right) \frac{\partial}{\partial y}\right]^{2 k-1} f\left(t, \alpha_{0}(t), \max _{s \in[t-h, t]} \alpha_{0}(s)\right) \\
\equiv & G_{0}\left(t, \alpha_{1}(t), \max _{s \in[t-h, t]} \alpha_{1}(s)\right), t \in[0, T], \\
\alpha_{0}(t) \leqslant & \varphi(t)+p_{0}^{2 k-1} C_{0}, t \in[-h, 0],
\end{aligned}
$$

and

$$
\begin{aligned}
A \beta_{0}^{\prime}(t) & \geqslant f\left(t, \beta_{0}(t), \max _{s \in[t-h, t]} \beta_{0}(s)\right)=G_{0}\left(t, \beta_{0}(t), \max _{s \in[t-h, t]} \beta_{0}(s)\right), t \in[0, T], \\
\beta_{0}(t) & \geqslant \varphi(t)+p_{0}^{2 k-1} C_{0}, t \in[-h, 0] .
\end{aligned}
$$

Furthermore, by Lemma 2.5, we see that there exists a solution $\beta_{1}(t)$ of IVP (3.14) such that $\alpha_{1}(t) \leqslant$ $\beta_{1}(t) \leqslant \beta_{0}(t)$ on $[-h, T]$.

Next, we must show that $\alpha_{1}(t)$ and $\beta_{1}(t)$ are lower and upper solutions of IVP (2.1), respectively. From the fact that $\alpha_{1}(t)$ is a solution of IVP (3.13) and the inequality (3.11), we get

$$
A \alpha_{1}^{\prime}(t) \leqslant f\left(t, \alpha_{1}(t), \max _{s \in[t-h, t]} \alpha_{1}(s)\right), \quad t \in[0, T], \quad \alpha_{1}(t) \leqslant \varphi(t), \quad t \in[-h, 0] .
$$

Hence, $\alpha_{1}(t)$ is a lower solution of IVP $(2.1)$ on $[-h, T]$. Similarly, using the conclusion that $\beta_{1}(t)$ is a solution of IVP (3.14) and the condition $\left(\mathrm{A}_{3.3}\right)$, we obtain

$$
\begin{aligned}
A \beta_{1}^{\prime}(t)= & f\left(t, \beta_{1}(t), \max _{s \in[t-h, t]} \beta_{1}(s)\right)-\frac{1}{(2 k-1) !} \int_{0}^{1}\left[\left(\beta_{1}(t)-\beta_{0}(t)\right) \frac{\partial}{\partial x}\right. \\
& \left.+\left(\max _{s \in[t-h, t]} \beta_{1}(s)-\max _{s \in[t-h, t]} \beta_{0}(s)\right) \frac{\partial}{\partial y}\right]^{2 k-1} \\
& \times f\left(t, \sigma \beta_{1}(t)+(1-\sigma) \beta_{0}(t), \sigma \max _{s \in[t-h, t]} \beta_{1}(s)+(1-\sigma) \max _{s \in[t-h, t]} \beta_{0}(s)\right) d \sigma
\end{aligned}
$$




$$
\begin{aligned}
& +\frac{1}{(2 k-1) !}\left[\left(\beta_{1}(t)-\beta_{0}(t)\right) \frac{\partial}{\partial x}\right. \\
& \left.+\left(\max _{s \in[t-h, t]} \beta_{1}(s)-\max _{s \in[t-h, t]} \beta_{0}(s)\right) \frac{\partial}{\partial y}\right]^{2 k-1} f\left(t, \alpha_{0}(t), \max _{s \in[t-h, t]} \alpha_{0}(s)\right) \\
\geqslant & f\left(t, \beta_{1}(t), \max _{s \in[t-h, t]} \beta_{1}(s)\right), t \in[0, T], \\
\beta_{1}(t) \geqslant & \varphi(t), \quad t \in[-h, 0] .
\end{aligned}
$$

This shows that $\beta_{1}(t)$ is an upper solution of IVP (2.1) on $[-h, T]$. Therefore, it follows that

$$
\alpha_{0}(t) \leqslant \alpha_{1}(t) \leqslant \beta_{1}(t) \leqslant \beta_{0}(t), \quad t \in[-h, T] .
$$

By induction, we can get that

$$
\alpha_{0}(t) \leqslant \alpha_{1}(t) \leqslant \cdots \leqslant \alpha_{n+1}(t) \leqslant \beta_{n+1}(t) \leqslant \cdots \leqslant \beta_{1}(t) \leqslant \beta_{0}(t), \quad t \in[-h, T],
$$

where $\alpha_{n+1}(t)$ and $\beta_{n+1}(t)$ are the solutions of the following singular differential systems

$$
\left\{\begin{aligned}
A x^{\prime}(t)= & f\left(t, \alpha_{n}(t), \max _{s \in[t-h, t]} \alpha_{n}(s)\right)+\sum_{i=1}^{2 k-1} \frac{1}{i !}\left[\left(x(t)-\alpha_{n}(t)\right) \frac{\partial}{\partial x}\right. \\
& \left.+\left(\max _{s \in[t-h, t]} x(s)-\max _{s \in[t-h, t]} \alpha_{n}(s)\right) \frac{\partial}{\partial y}\right]^{i} f\left(t, \alpha_{n}(t), \max _{s \in[t-h, t]} \alpha_{n}(s)\right) \\
\equiv & F_{n}\left(t, x(t), \max _{s \in[t-h, t]} x(s)\right), t \in[0, T] \\
x(t)= & \varphi(t)-k_{n}^{2 k-1} L_{n}, t \in[-h, 0]
\end{aligned}\right.
$$

and

$$
\left\{\begin{aligned}
A x^{\prime}(t)= & f\left(t, \beta_{n}(t), \max _{s \in[t-h, t]} \beta_{n}(s)\right)+\sum_{i=1}^{2 k-2} \frac{1}{i !}\left[\left(x(t)-\beta_{n}(t)\right) \frac{\partial}{\partial x}\right. \\
& \left.+\left(\max _{s \in[t-h, t]} x(s)-\max _{s \in[t-h, t]} \beta_{n}(s)\right) \frac{\partial}{\partial y}\right]^{i} f\left(t, \beta_{n}(t), \max _{s \in[t-h, t]} \beta_{n}(s)\right) \\
& +\frac{1}{(2 k-1) !}\left[\left(x(t)-\beta_{n}(t)\right) \frac{\partial}{\partial x}\right. \\
& \left.+\left(\max _{s \in[t-h, t]} x(s)-\max _{s \in[t-h, t]} \beta_{n}(s)\right) \frac{\partial}{\partial y}\right]^{2 k-1} f\left(t, \alpha_{n}(t), \max _{s \in[t-h, t]} \alpha_{n}(s)\right) \\
\equiv & G_{n}\left(t, x(t), \max _{s \in[t-h, t]} x(s)\right), t \in[0, T], \\
x(t)= & \varphi(t)+p_{n}^{2 k-1} C_{n}, t \in[-h, 0] .
\end{aligned}\right.
$$

Employing the Ascoli-Arzelà Theorem, both monotone sequences $\left\{\alpha_{n}(t)\right\}$ and $\left\{\beta_{\mathfrak{n}}(t)\right\}$ converge uniformly to the solution of IVP (2.1).

Finally, we prove that the convergence of the sequences $\left\{\alpha_{n}(t)\right\}$ and $\left\{\beta_{n}(t)\right\}$ to the solution $x(t)$ of IVP (2.1) is of order $2 k$. To do this, let

$$
a_{n+1}(t)=x(t)-\alpha_{n+1}(t) \geqslant 0, \quad t \in[-h, T] .
$$

The proof is identical to Theorem 3.1. By comparing the IVPs (3.5) and (3.15), we get

$$
\left|a_{n+1}\right|_{0} \leqslant K_{3}\left|a_{n}\right|_{0}^{2 k}
$$

where $K_{3}$ is a positive matrix. Therefore, the convergence of the sequence $\left\{\alpha_{n}(t)\right\}$ is of order $2 k$.

On the other hand, consider

$$
b_{n+1}(t)=\beta_{n+1}(t)-x(t) \geqslant 0, \quad t \in[-h, T] .
$$


Case 1. If $t \in[-h, 0]$, since $\beta_{n+1}(t)-x(t)=p_{n}^{2 k-1} C_{n}$ and $p_{n i} \leqslant C_{n i}$, we have

$$
\beta_{n+1}(t)-x(t)=p_{n}^{2 k-1} C_{n} \leqslant\left(C_{n}\right)^{2 k}=\left(\min _{s \in[-h, 0]}\left(\beta_{n}(s)-x(s)\right)\right)^{2 k} \leqslant \max _{s \in[-h, 0]}\left|\beta_{n}(s)-x(s)\right|^{2 k},
$$

which implies that

$$
b_{n+1}(t) \leqslant \max _{s \in[-h, 0]}\left|b_{n}(s)\right|^{2 k}
$$

Case 2. If $t \in[0, T]$, using the condition $\left(\mathrm{A}_{3.3}\right)$ and the mean value theorem, we obtain

$$
\begin{aligned}
& A b_{n+1}^{\prime}(t)=f\left(t, \beta_{n}(t), \max _{s \in[t-h, t]} \beta_{n}(s)\right)+\sum_{i=1}^{2 k-2} \frac{1}{i !}\left[\left(\beta_{n+1}(t)-\beta_{n}(t)\right) \frac{\partial}{\partial x}\right. \\
& \left.+\left(\max _{s \in[t-h, t]} \beta_{n+1}(s)-\max _{s \in[t-h, t]} \beta_{n}(s)\right) \frac{\partial}{\partial y}\right]^{i} f\left(t, \beta_{n}(t), \max _{s \in[t-h, t]} \beta_{n}(s)\right) \\
& +\frac{1}{(2 k-1) !}\left[\left(\beta_{n+1}(t)-\beta_{n}(t)\right) \frac{\partial}{\partial x}+\left(\max _{s \in[t-h, t]} \beta_{n+1}(s)-\max _{s \in[t-h, t]} \beta_{n}(s)\right) \frac{\partial}{\partial y}\right]^{2 k-1} \\
& \times f\left(t, \alpha_{n}(t), \max _{s \in[t-h, t]} \alpha_{n}(s)\right)-f\left(t, x(t), \max _{s \in[t-h, t]} x(s)\right) \\
& =f\left(t, \beta_{n+1}(t), \max _{s \in[t-h, t]} \beta_{n+1}(s)\right)-\frac{1}{(2 k-1) !} \int_{0}^{1}\left[\left(\beta_{n+1}(t)-\beta_{n}(t)\right) \frac{\partial}{\partial x}\right. \\
& \left.+\left(\max _{s \in[t-h, t]} \beta_{n+1}(s)-\max _{s \in[t-h, t]} \beta_{n}(s)\right) \frac{\partial}{\partial y}\right]^{2 k-1} \\
& \times f\left(t, \sigma \beta_{n+1}(t)+(1-\sigma) \beta_{n}(t), \sigma \max _{s \in[t-h, t]} \beta_{n+1}(s)+(1-\sigma) \max _{s \in[t-h, t]} \beta_{n}(s)\right) d \sigma \\
& +\frac{1}{(2 k-1) !}\left[\left(\beta_{n+1}(t)-\beta_{n}(t)\right) \frac{\partial}{\partial x}+\left(\max _{s \in[t-h, t]} \beta_{n+1}(s)-\max _{s \in[t-h, t]} \beta_{n}(s)\right) \frac{\partial}{\partial y}\right]^{2 k-1} \\
& \times f\left(t, \alpha_{n}(t), \max _{s \in[t-h, t]} \alpha_{n}(s)\right)-f\left(t, x(t), \max _{s \in[t-h, t]} x(s)\right) \\
& \leqslant\left(\int_{0}^{1} f_{x}\left(t, \sigma \beta_{n+1}(t)+(1-\sigma) x(t), \max _{s \in[t-h, t]} \beta_{n+1}(s)\right) d \sigma\right)\left(\beta_{n+1}(t)-x(t)\right) \\
& +\left(\int_{0}^{1} f_{y}\left(t, x(t), \sigma \max _{s \in[t-h, t]} \beta_{n+1}(s)+(1-\sigma) \max _{s \in[t-h, t]} \beta_{n}(s)\right) d \sigma\right) \\
& \times \max _{s \in[t-h, t]}\left(\beta_{n+1}(s)-x(s)\right) \\
& +2^{2 k} M_{2} \max _{s \in[t-h, t]}\left|b_{n}(s)\right|^{2 k-1}\left(\max _{s \in[t-h, t]}\left|b_{n}(s)\right|+\max _{s \in[t-h, t]}\left|a_{n}(s)\right|\right) \\
& =-M(t) b_{n+1}(t)-N(t) \max _{s \in[t-h, t]} b_{n+1}(s) \\
& +2^{2 k} M_{2} \max _{s \in[t-h, t]}\left|b_{n}(s)\right|^{2 k-1}\left(\max _{s \in[t-h, t]}\left|b_{n}(s)\right|+\max _{s \in[t-h, t]}\left|a_{n}(s)\right|\right) \\
& \leqslant-M(t) b_{n+1}(t)-N(t) \max _{s \in[-h, T]} b_{n+1}(s) \\
& +2^{2 \mathrm{k}} \mathrm{M}_{2} \max _{s \in[-\mathrm{h}, \mathrm{T}]}\left|\mathrm{b}_{\mathrm{n}}(\mathrm{s})\right|^{2 \mathrm{k}-1}\left(\max _{\mathrm{s} \in[-\mathrm{h}, \mathrm{T}]}\left|\mathrm{b}_{\mathrm{n}}(\mathrm{s})\right|+\max _{\mathrm{s} \in[-\mathrm{h}, \mathrm{T}]}\left|\mathrm{a}_{\mathrm{n}}(\mathrm{s})\right|\right),
\end{aligned}
$$

where $\frac{1}{(2 k-1) !}\left[\left(\beta_{n}(t)-x(t)\right) \frac{\partial}{\partial x}+\left(\max _{s \in[t-h, t]} \beta_{n}(s)-\max _{s \in[t-h, t]} x(s)\right) \frac{\partial}{\partial y}\right]^{2 k-1}\left(f\left(t, \beta_{n}(t), \max _{s \in[t-h, t]} \beta_{n}(s)\right)-\right.$ $\left.f\left(t, \alpha_{n}(t), \max _{s \in[t-h, t]} \alpha_{n}(s)\right)\right) \leqslant 2^{2 k} M_{2}\left(\max _{s \in[t-h, t]} b_{n}(s)\right)^{2 k-1}\left(\max _{s \in[t-h, t]} b_{n}(s)+\max _{s \in[t-h, t]} a_{n}(s)\right)$, and $M_{2}$ is a positive $n \times n$ matrix. By Lemma 2.2, we can get that $b_{n+1}(t) \leqslant u(t)$ on $[0, T]$, where $u(t)$ is the solution 
of

$$
\begin{aligned}
A u^{\prime}(t)+M(t) u(t)= & -N(t) \max _{s \in[-h, T]} b_{n+1}(s)+2{ }^{2 k} M_{2} \max _{s \in[-h, T]}\left|b_{n}(s)\right|^{2 k-1}\left(\max _{s \in[-h, T]}\left|b_{n}(s)\right|\right. \\
& \left.+\max _{s \in[-h, T]}\left|a_{n}(s)\right|\right), \quad t \in[0, T], \quad u(t)=p_{n}^{2 k-1} C_{n}, \quad t \in[-h, 0] .
\end{aligned}
$$

Thus, using the expression of $x(t)$ in Lemma 2.3, we obtain

$$
\begin{aligned}
& \max _{s \in[-h, T]} b_{n+1}(s) \\
& \leqslant\left\{I-\max _{s \in[0, T]}\left\{-[\lambda A+M(s)]^{-1}\left[e^{-\hat{A}^{D} \hat{M} s} \int_{0}^{s} e^{\hat{A}^{D} \hat{M} \sigma} \hat{A}^{D} N(\sigma) d \sigma\right.\right.\right. \\
&\left.\left.\left.+\left(I-\hat{A} \hat{A}^{D}\right) \hat{M}^{D} N(s)\right]\right\}\right\}^{-1} \max _{s \in[0, T]}\left\{[ \lambda A + M ( s ) ] ^ { - 1 } \left[e^{-\hat{A}^{D} \hat{M} s} \hat{A} \hat{A}^{D}[\lambda A+M(s)] k_{n}^{2 k} L_{n}\right.\right. \\
&+e^{-\hat{A}^{D} \hat{M} s} \int_{0}^{s} e^{\hat{A}^{D} \hat{M} \sigma} \hat{A}^{D} 2^{2 k} M_{2} \max _{s \in[-h, T]}\left|b_{n}(s)\right|^{2 k-1}\left(\max _{s \in[-h, T]}\left|b_{n}(s)\right|+\max _{s \in[-h, T]}\left|a_{n}(s)\right|\right) d \sigma \\
&\left.\left.+\left(I-\hat{A} \hat{A}^{D}\right) \hat{M}^{D} 2^{2 k} M_{2} \max _{s \in[-h, T]}\left|b_{n}(s)\right|^{2 k-1}\left(\max _{s \in[-h, T]}\left|b_{n}(s)\right|+\max _{s \in[-h, T]}\left|a_{n}(s)\right|\right)\right]\right\} .
\end{aligned}
$$

Therefore, from the inequalities (3.17) and (3.18), and making suitable estimates, we have

$$
\left|b_{n+1}\right|_{0} \leqslant K_{4}\left|b_{n}\right|_{0}^{2 k-1}\left(\left|b_{n}\right|_{0}+\left|a_{n}\right|_{0}\right),
$$

where $K_{4}$ is a positive matrix. This shows that the convergence of the sequence $\left\{\beta_{n}(t)\right\}$ is of order $2 k$. The proof is complete.

Theorem 3.3. Assume that the condition $\left(\mathrm{A}_{3.2}\right)$ holds, and

$\left(A_{3.4}\right)$ there exists a function $f \in C^{0,2 k+1,2 k+1}\left(\Omega\left(\alpha_{0}, \beta_{0}\right), R^{n}\right)$ such that the Fréchet derivatives $f_{y}(t, x, y) \geqslant 0$, $\frac{\partial^{2 k+1} f(t, x, y)}{\partial x^{i} y^{2 k+1-i}} \leqslant 0$ for $k \geqslant 1, i=0,1,2, \cdots, 2 k+1$.

Then there exist two sequences $\left\{\alpha_{\mathrm{n}}(\mathrm{t})\right\}$ and $\left\{\beta_{\mathrm{n}}(\mathrm{t})\right\}$ converging uniformly to the solution $\mathrm{x}(\mathrm{t})$ of IVP (2.1) and the convergence is of order $2 \mathrm{k}+1$, that is, there exist positive matrices $\mathrm{K}_{5}, \mathrm{~K}_{6}$ such that for the solution $\mathrm{x}(\mathrm{t})$ of IVP (2.1) in $\mathrm{S}\left(\alpha_{0}, \beta_{0}\right)$, the inequalities

$$
\left|x-\alpha_{n+1}\right|_{0} \leqslant K_{5}\left|x-\alpha_{n}\right|_{0}^{2 k}\left(\left|x-\alpha_{n}\right|_{0}+\left|\beta_{n}-x\right|_{0}\right), \quad\left|\beta_{n+1}-x\right|_{0} \leqslant K_{6}\left|\beta_{n}-x\right|_{0}^{2 k}\left(\left|\beta_{n}-x\right|_{0}+\left|x-\alpha_{n}\right|_{0}\right)
$$

hold, where $|\mathfrak{u}|_{0}=\max _{s \in[-h, T]}|\mathfrak{u}(s)|=\left(\max _{s \in[-h, T]}\left|u_{1}(s)\right|, \max _{s \in[-h, T]}\left|u_{2}(s)\right|, \cdots, \max _{s \in[-h, T]}\left|u_{n}(s)\right|\right)^{\top}$.

Proof. In order to construct the monotone sequences $\left\{\alpha_{n}(t)\right\},\left\{\beta_{n}(t)\right\}$, and obtain the rapid convergence, we consider the following singular differential systems with "maxima"

$$
\left\{\begin{aligned}
A x^{\prime}(t)= & f\left(t, \alpha_{n}(t), \max _{s \in[t-h, t]} \alpha_{n}(s)\right)+\sum_{i=1}^{2 k-1} \frac{1}{i !}\left[\left(x(t)-\alpha_{n}(t)\right) \frac{\partial}{\partial x}\right. \\
& \left.+\left(\max _{s \in[t-h, t]} x(s)-\max _{s \in[t-h, t]} \alpha_{n}(s)\right) \frac{\partial}{\partial y}\right]^{i} f\left(t, \alpha_{n}(t), \max _{s \in[t-h, t]} \alpha_{n}(s)\right) \\
& +\frac{1}{(2 k) !}\left[\left(x(t)-\alpha_{n}(t)\right) \frac{\partial}{\partial x}\right. \\
& \left.+\left(\max _{s \in[t-h, t]} x(s)-\max _{s \in[t-h, t]} \alpha_{n}(s)\right) \frac{\partial}{\partial y}\right]^{2 k} f\left(t, \beta_{n}(t), \max _{s \in[t-h, t]} \beta_{n}(s)\right) \\
\equiv & F_{n}\left(t, x(t), \max _{s \in[t-h, t]} x(s)\right), t \in[0, T], \\
x(t)= & \varphi(t)-k_{n}^{2 k} L_{n}, t \in[-h, 0],
\end{aligned}\right.
$$


and

$$
\left\{\begin{aligned}
A x^{\prime}(t)= & f\left(t, \beta_{n}(t), \max _{s \in[t-h, t]} \beta_{n}(s)\right)+\sum_{i=1}^{2 k-1} \frac{1}{i !}\left[\left(x(t)-\beta_{n}(t)\right) \frac{\partial}{\partial x}\right. \\
& \left.+\left(\max _{s \in[t-h, t]} x(s)-\max _{s \in[t-h, t]} \beta_{n}(s)\right) \frac{\partial}{\partial y}\right]^{i} f\left(t, \beta_{n}(t), \max _{s \in[t-h, t]} \beta_{n}(s)\right) \\
& +\frac{1}{(2 k) !}\left[\left(x(t)-\beta_{n}(t)\right) \frac{\partial}{\partial x}\right. \\
& \left.+\left(\max _{s \in[t-h, t]} x(s)-\max _{s \in[t-h, t]} \beta_{n}(s)\right) \frac{\partial}{\partial y}\right]^{2 k} f\left(t, \alpha_{n}(t), \max _{s \in[t-h, t]} \alpha_{n}(s)\right) \\
\equiv & G_{n}\left(t, x(t), \max _{s \in[t-h, t]} x(s)\right), t \in[0, T], \\
x(t)= & \varphi(t)+p_{n}^{2 k} C_{n}, t \in[-h, 0] .
\end{aligned}\right.
$$

The proceeding of proof is similar to Theorem 3.1, and thus we omit the details.

Theorem 3.4. Assume that the condition $\left(\mathrm{A}_{3.2}\right)$ holds, and

( $\left.\mathrm{A}_{3.5}\right)$ there exists a function $\mathrm{f} \in \mathrm{C}^{0,2 \mathrm{k}, 2 \mathrm{k}}\left(\Omega\left(\alpha_{0}, \beta_{0}\right), \mathrm{R}^{\mathrm{n}}\right)$ such that the Fréchet derivatives $\mathrm{f}_{\mathrm{y}}(\mathrm{t}, \mathrm{x}, \mathrm{y}) \geqslant 0$, $\frac{\partial^{2 k} f(t, x, y)}{\partial x^{i} y^{2 k-i}} \leqslant 0$ for $k \geqslant 1, i=0,1,2, \cdots, 2 k$.

Then there exist two sequences $\left\{\alpha_{n}(t)\right\}$ and $\left\{\beta_{n}(t)\right\}$ converging uniformly to the solution $x(t)$ of IVP (2.1) and the convergence is of order $2 \mathrm{k}$, that is, there exist positive matrices $\mathrm{K}_{7}, \mathrm{~K}_{8}$ such that for the solution $\mathrm{x}(\mathrm{t})$ of IVP (2.1) in $S\left(\alpha_{0}, \beta_{0}\right)$, the inequalities

$$
\left|x-\alpha_{n+1}\right|_{0} \leqslant K_{7}\left|x-\alpha_{n}\right|_{0}^{2 k-1}\left(\left|x-\alpha_{n}\right|_{0}+\left|\beta_{n}-x\right|_{0}\right), \quad\left|\beta_{n+1}-x\right|_{0} \leqslant K_{8}\left|\beta_{n}-x\right|_{0}^{2 k}
$$

hold, where $|\mathfrak{u}|_{0}=\max _{s \in[-h, T]}|\mathfrak{u}(s)|=\left(\max _{s \in[-h, T]}\left|u_{1}(s)\right|, \max _{s \in[-h, T]}\left|u_{2}(s)\right|, \cdots, \max _{s \in[-h, T]}\left|u_{n}(s)\right|\right)^{\top}$.

Proof. To construct the monotone sequences $\left\{\alpha_{n}(t)\right\},\left\{\beta_{n}(t)\right\}$, and obtain the rapid convergence, consider the following singular differential systems with "maxima"

$$
\left\{\begin{aligned}
A x^{\prime}(t)= & f\left(t, \alpha_{n}(t), \max _{s \in[t-h, t]} \alpha_{n}(s)\right)+\sum_{i=1}^{2 k-2} \frac{1}{i !}\left[\left(x(t)-\alpha_{n}(t)\right) \frac{\partial}{\partial x}\right. \\
& \left.+\left(\max _{s \in[t-h, t]} x(s)-\max _{s \in[t-h, t]} \alpha_{n}(s)\right) \frac{\partial}{\partial y}\right]^{i} f\left(t, \alpha_{n}(t), \max _{s \in[t-h, t]} \alpha_{n}(s)\right) \\
& +\frac{1}{(2 k-1) !}\left[\left(x(t)-\alpha_{n}(t)\right) \frac{\partial}{\partial x}\right. \\
& \left.+\left(\max _{s \in[t-h, t]} x(s)-\max _{s \in[t-h, t]} \alpha_{n}(s)\right) \frac{\partial}{\partial y}\right]^{2 k-1} f\left(t, \beta_{n}(t), \max _{s \in[t-h, t]} \beta_{n}(s)\right) \\
\equiv & F_{n}\left(t, x(t), \max _{s \in[t-h, t]} x(s)\right), t \in[0, T], \\
x(t)= & \varphi(t)-k_{n}^{2 k-1} L_{n}, t \in[-h, 0],
\end{aligned}\right.
$$

and

$$
\left\{\begin{aligned}
A x^{\prime}(t)= & f\left(t, \beta_{n}(t), \max _{s \in[t-h, t]} \beta_{n}(s)\right)+\sum_{i=1}^{2 k-1} \frac{1}{i !}\left[\left(x(t)-\beta_{n}(t)\right) \frac{\partial}{\partial x}\right. \\
& \left.+\left(\max _{s \in[t-h, t]} x(s)-\max _{s \in[t-h, t]} \beta_{n}(s)\right) \frac{\partial}{\partial y}\right]^{i} f\left(t, \beta_{n}(t), \max _{s \in[t-h, t]} \beta_{n}(s)\right) \\
\equiv & G_{n}\left(t, x(t), \max _{s \in[t-h, t]} x(s)\right), t \in[0, T], \\
x(t)= & \varphi(t)+p_{n}^{2 k-1} C_{n}, t \in[-h, 0] .
\end{aligned}\right.
$$

The proceeding of proof is similar to Theorem 3.1, and thus we omit the details. 


\section{Example}

Now, we give an example to illustrate the application of the established in the previous section.

Example 4.1. Consider the following singular differential system with "maxim"

$$
\left\{\begin{aligned}
x_{1}^{\prime}(t) & =-x_{1}(t)+\max _{s \in[t-h, t]} x_{1}(s)+\frac{1}{1-\max _{s \in[t-h, t]} x_{1}(s)}-1, \\
0 & =\frac{1}{1-x_{2}(t)}+\frac{1}{1-\max _{s \in[t-h, t]} x_{2}(s)}-2, \quad t \in[0,1], \\
x_{1}(t) & =x_{2}(t)=0, \quad t \in[-1,0],
\end{aligned}\right.
$$

where

$$
A=\left(\begin{array}{ll}
1 & 0 \\
0 & 0
\end{array}\right), f\left(t, x(t), \max _{s \in[t-h, t]} x(s)\right)=\left(\begin{array}{c}
-x_{1}(t)+\max _{s \in[t-h, t]} x_{1}(s)+\frac{1}{1-\max _{s \in[t-h, t]} x_{1}(s)}-1 \\
\frac{1}{1-x_{2}(t)}+\frac{1}{1-\max _{s \in[t-h, t]} x_{2}(s)}-2
\end{array}\right) .
$$

One can see easily that the IVP (4.1) has zero solution. Taking $\alpha_{0}(t)=(0,0)^{\top}, \beta_{0}(t)=\left(\frac{1}{2}, \frac{1}{2}\right)^{\top}$, it is easy to verify that $\alpha_{0}(t), \beta_{0}(t)$ are lower and upper solutions of IVP (4.1), respectively, and $f_{y} \geqslant 0, \frac{\partial^{2} f(t, x, y)}{\partial x^{2}}$, $\frac{\partial^{2} f(t, x, y)}{\partial x \partial y}, \frac{\partial^{2} f(t, x, y)}{\partial y^{2}} \geqslant 0$ on $\Omega\left(\alpha_{0}, \beta_{0}\right)$, that is, the conditions of Theorem 3.2 are satisfied. According to Theorem 3.2, we can construct two monotone sequences of functions which quadratically converge to the zero solution of IVP (4.1).

The successive approximations $\alpha_{n+1}(t)$ and $\beta_{n+1}(t)$ are solutions of the singular differential systems

$$
\left\{\begin{aligned}
x_{1}^{\prime}(t)= & -\alpha_{n 1}(t)+\max _{s \in[t-h, t]} \alpha_{n 1}(s)+\frac{1}{1-\max _{s \in[t-h, t]} \alpha_{n 1}(s)}-1 \\
& -\left(x_{1}(t)-\alpha_{n 1}(t)\right)+\left(\max _{s \in[t-h, t]} x_{1}(s)-\max _{s \in[t-h, t]} \alpha_{n 1}(s)\right) \\
& +\frac{1}{\left(1-\max _{s \in[t-h, t]} \beta_{n 1}(s)\right)^{2}}\left(\max _{s \in[t-h, t]} x_{1}(s)-\max _{s \in[t-h, t]} \alpha_{n 1}(s)\right), \\
0= & \frac{1}{1-\alpha_{n 2}(t)}+\frac{1}{1-\max _{s \in[t-h, t]} \alpha_{n 2}(s)}-2+\frac{1}{\left(1-\beta_{n 2}(t)\right)^{2}}\left(x_{2}(t)-\alpha_{n 2}(t)\right) \\
& +\frac{1}{\left(1-\max _{s \in[t-h, t]} \beta_{n 2}(s)\right)^{2}}\left(\max _{s \in[t-h, t]} x_{2}(s)-\max _{s \in[t-h, t]} \alpha_{n 2}(s)\right), \quad t \in[0,1], \\
x_{1}(t)= & x_{2}(t)=-k_{n 1} L_{n 1}=-k_{n 2} L_{n 2}, t \in[-1,0],
\end{aligned}\right.
$$

and

$$
\left\{\begin{aligned}
x_{1}^{\prime}(t)= & -\beta_{n 1}(t)+\max _{s \in[t-h, t]} \beta_{n 1}(s)+\frac{1}{1-\max _{s \in[t-h, t]} \beta_{n 1}(s)}-1 \\
& -\left(x_{1}(t)-\beta_{n 1}(t)\right)+\left(\max _{s \in[t-h, t]} x_{1}(s)-\max _{s \in[t-h, t]} \beta_{n 1}(s)\right) \\
& +\frac{1}{\left(1-\max _{s \in[t-h, t]} \beta_{n 1}(s)\right)^{2}}\left(\max _{s \in[t-h, t]} x_{1}(s)-\max _{s \in[t-h, t]} \beta_{n 1}(s)\right), \\
0= & \frac{1}{1-\beta_{n 2}(t)}+\frac{1}{1-\max _{s \in[t-h, t]} \beta_{n 2}(s)}-2+\frac{1}{\left(1-\beta_{n 2}(t)\right)^{2}}\left(x_{2}(t)-\beta_{n 2}(t)\right) \\
& +\frac{1}{\left(1-\max _{s \in[t-h, t]} \beta_{n 2}(s)\right)^{2}}\left(\max _{s \in[t-h, t]} x_{2}(s)-\max _{s \in[t-h, t]} \beta_{n 2}(s)\right), \quad t \in[0,1], \\
x_{1}(t)= & x_{2}(t)=p_{n 1} C_{n 1}=p_{n 2} C_{n 2}, \quad t \in[-1,0],
\end{aligned}\right.
$$


where $L_{n}=\min _{s \in[-1,0]}\left(-\alpha_{n}(s)\right) \geqslant 0, C_{n}=\min _{s \in[-1,0]}\left(\beta_{n}(s)\right) \geqslant 0$, and the numbers $k_{n i}, p_{n i} \in[0,1)$ are such that $k_{n i} \leqslant L_{n i}$ and $p_{n i} \leqslant C_{n i}, i=1,2, \cdots, n$.

First, we will construct an increasing sequence of lower solutions which converges quadratically to the zero solution. In view of the fact that $\alpha_{0}(t)=(0,0)^{\mathrm{T}}$, we have $\mathrm{L}_{0}=(0,0)^{\mathrm{T}}$. Taking $\mathrm{k}_{0}=(0,0)^{\mathrm{T}}$, the linear singular differential system (4.2) reduces to

$$
\left\{\begin{array}{l}
x_{1}^{\prime}(t)=-x_{1}(t)+5 \max _{s \in[t-h, t]} x_{1}(s) \\
0=4 x_{2}(t)+4 \max _{s \in[t-h, t]} x_{2}(s), t \in[0,1] \\
x_{1}(t)=x_{2}(t)=0, \quad t \in[-1,0]
\end{array}\right.
$$

Then, the IVP (4.4) has a zero solution, that is, $\alpha_{1}(t)=(0,0)^{\top}$. Proceeding as before, we can find that all successive approximations $\alpha_{n}(t)$ are equal to the zero vector.

Next, we shall construct a decreasing sequence of upper solutions which will converge quadratically to the exact solution. Since $\beta_{0}(t)=\left(\frac{1}{2}, \frac{1}{2}\right)^{\mathrm{T}}$, we get $C_{0}=\left(\frac{1}{2}, \frac{1}{2}\right)^{\mathrm{T}}$. Let $p_{0}=\left(\frac{1}{2}, \frac{1}{2}\right)^{\mathrm{T}}$, then the linear singular differential system (4.3) can be written as

$$
\left\{\begin{aligned}
x_{1}^{\prime}(t) & =-x_{1}(t)+5 \max _{s \in[t-h, t]} x_{1}(s)-1 \\
0 & =4 x_{2}(t)+4 \max _{s \in[t-h, t]} x_{2}(s)-2, t \in[0,1] \\
x_{1}(t) & =x_{2}(t)=\frac{1}{2^{2}}, \quad t \in[-1,0]
\end{aligned}\right.
$$

The function $\beta_{1}(t)=\left(\frac{1}{4}, \frac{1}{4}\right)^{\top}$ is a solution of IVP (4.5).

Choose $p_{1}=\left(\frac{1}{4}, \frac{1}{4}\right)^{\top}$, then the linear singular differential system (4.3) reduces to the following

$$
\left\{\begin{aligned}
x_{1}^{\prime}(t) & =-x_{1}(t)+\frac{25}{9} \max _{s \in[t-h, t]} x_{1}(s)-\frac{1}{9} \\
0 & =\frac{16}{9} x_{2}(t)+\frac{16}{9} \max _{s \in[t-h, t]} x_{2}(s)-\frac{2}{9}, \quad t \in[0,1] \\
x_{1}(t) & =x_{2}(t)=\frac{1}{4^{2}}, \quad t \in[-1,0]
\end{aligned}\right.
$$

The IVP (4.6) has an exact solution $\beta_{2}(t)=\left(\frac{1}{16}, \frac{1}{16}\right)^{\mathrm{T}}$.

Now, choose $p_{n-1}=\left(\frac{1}{2^{2^{(n-1)}}}, \frac{1}{2^{2^{(n-1)}}}\right)^{\top}$, then the linear singular differential system (4.3) reduces to

$$
\left\{\begin{aligned}
x_{1}^{\prime}(t) & =-x_{1}(t)+\left(1+\frac{2^{2^{n}}}{\left(2^{2^{(n-1)}}-1\right)^{2}}\right) \max _{s \in[t-h, t]} x_{1}(s)-\frac{1}{\left(2^{2^{(n-1)}}-1\right)^{2}} \\
0 & =\frac{2^{2^{n}}}{\left(2^{2^{(n-1)}}-1\right)^{2}} x_{2}(t)+\frac{2^{2^{n}}}{\left(2^{2^{(n-1)}}-1\right)^{2}} \max _{s \in[t-h, t]} x_{2}(s)-\frac{2}{\left(2^{2^{(n-1)}}-1\right)^{2}}, t \in[0,1], \\
x_{1}(t) & =x_{2}(t)=\frac{1}{2^{2^{n}}}, t \in[-1,0] .
\end{aligned}\right.
$$

Then, the IVP (4.7) has an exact solution $\beta_{\mathfrak{n}}(t)=\left(\frac{1}{2^{2^{n}}}, \frac{1}{2^{2^{n}}}\right)^{\mathrm{T}}$. that

By induction, we obtain $\beta_{1}(t)=\left(\frac{1}{4}, \frac{1}{4}\right)^{\top}, \beta_{2}(t)=\left(\frac{1}{16}, \frac{1}{16}\right)^{\top}, \ldots, \beta_{\mathfrak{n}}(t)=\left(\frac{1}{2^{2^{n}}}, \frac{1}{2^{2^{n}}}\right)^{\top}, \ldots$. It is easy to see

$$
\left(\begin{array}{c}
\frac{1}{4} \\
\frac{1}{4}
\end{array}\right) \leqslant\left(\begin{array}{ll}
1 & 1 \\
1 & 1
\end{array}\right)\left(\begin{array}{c}
\frac{1}{2^{2}} \\
\frac{1}{2^{2}}
\end{array}\right),\left(\begin{array}{c}
\frac{1}{16} \\
\frac{1}{16}
\end{array}\right) \leqslant\left(\begin{array}{cc}
1 & 1 \\
1 & 1
\end{array}\right)\left(\begin{array}{c}
\frac{1}{4^{2}} \\
\frac{1}{4^{2}}
\end{array}\right), \cdots,\left(\begin{array}{c}
\frac{1}{2^{2^{n}}} \\
\frac{1}{2^{2^{n}}}
\end{array}\right) \leqslant\left(\begin{array}{cc}
1 & 1 \\
1 & 1
\end{array}\right)\left(\begin{array}{c}
\frac{1}{\left(2^{2^{(n-1)}}\right)^{2}} \\
\frac{1}{\left(2^{2^{(n-1)}}\right)^{2}}
\end{array}\right), \cdots .
$$

Therefore, the convergence of the sequence $\left\{\beta_{n}(t)\right\}$ is quadratic. The proof is complete. 


\section{Acknowledgment}

This paper is supported by the National Natural Science Foundation of China (11771115 and 11271106$)$ and the Natural Science Foundation of Hebei Province of China (QN2017018).

\section{References}

[1] A. R. Abd-Ellateef Kamar, G. M. Attia, K. Vajravelu, M. Mosaad, Generlized quasilinearization for singular system of differential equations, Appl. Math. Comput., 114 (2000), 69-74. 1

[2] R. P. Agarwal, S. Hristova, Quasilinearization for initial value problems involving differential equations with "maxim", Math. Comput. modelling, 55 (2012), 2096-2105. 1

[3] B. Ahmad, A. Alsaedi, An extended method of quasilinearization for nonlinear impulsive differential equations with a nonlinear three-point boundary condition, Electron. J. Qual. Theory Differ. Equ., 2007 (2007), 19 pages. 1

[4] B. Ahmad, R. A. Khan, S. Sivasundaram, Generalized quasilinearization method for nonlinear functional differential equations, J. Appl. Math. Stochastic Anal., 16 (2003), 33-43. 1

[5] P. Amster, P. De Nápoli, A quasilinearization method for elliptic problems with a nonlinear boundary condition, Nonlinear Anal., 66 (2007), 2255-2263. 1

[6] D. D. Bainov, S. G. Hristova, The method of quasilinearization for the periodic boundary value problem for systems of impulsive differential equations, Appl. Math. Comput., 117 (2001), 73-85. 1

[7] D. D. Bainov, S. G. Hristova, Differential Equations with Maxima, CRC Press Taylor \& Francis, New York, (2011). 1

[8] R. E. Bellman, R. E. Kalaba, Quasilinearization and Nonlinear Boundary Value Problems, Elsevier, New York, (1965). 1

[9] A. Buică, Quasilinearization method for nonlinear elliptic boundary value problems, J. Optim. Theory Appl., 124 (2005), 323-338. 1

[10] S. L. Campbell, Singular systems of differential equations, Pitman Advanced Publishing Program (I), London, (1982). 1

[11] S. L. Campbell, Singular systems of differential equations, Pitman Advanced Publishing Program (II), London, (1982). $1,2.3$

[12] Z. Drici, F. A. McRae, J. V. Devi, Quasilinearization for functional differential equations with retardation and anticipation, Nonlinear Anal., 70 (2009), 1763-1775. 1

[13] M. A. El-Gebeily, D. O'Regan, Upper and lower solutions and quasilinearization for a class of second order singular nonlinear differential equations with nonlinear boundary conditions, Nonlinear Anal. Real World Appl., 8 (2007), 636645. 1

[14] M. A. El-Gebeily, D. O’Regan, Existence and quasilinearization for a class of nonlinear elliptic second order partial differential equations, Dynam. Systems Appl., 17 (2008), 445-458. 1

[15] S. Hristova, A. Golev, K. Stefanova, Quasilinearization of the initial value problem for difference equations with "maxim", J. Appl. Math., 2012 (2012), 17 pages. 1

[16] V. Lakshmikantham, S. Köksal, Monotone flows and rapid convergence for nonlinear partial differential equations, Taylor \& Francis, London, (2003). 1

[17] V. Laksmikantham, S. Leela, Z. Drici, F. A. McRae, Theory of causal differential equations, World Scientific, Hackensack, (2009). 1

[18] V. Lakshmikantham, A. S. Vatsala, Generalized quasilinearization for nonlinear problems, Kluwer Academic Publishers, Dordrecht, (1998). 1

[19] D. O'Regan, M. El-Gebeily, Existence, upper and lower solutions and quasilinearization for singular differential equations, IMA J. Appl. Math., 73 (2008), 323-344. 1

[20] H. H. Rosenbrock, Structural properties of linear dynamical systems, Internat. J. Control, 20 (1974), 191-202. 1

[21] J. Vasundhara Devi, F. A. McRae, Z. Drici, Generalized quasilinearization for fractional differential equations, Comput. Math. Appl., 59 (2010), 1057-1062. 1

[22] P.-G. Wang, W. Gao, Quasilinearization of an initial value problem for a set valued integro-differential equation, Comput. Math. Appl., 61 (2011), 2111-2115. 1

[23] P.-G. Wang, T.-T. Kong, Quasilinearization for the boundary value problem of second-order singular differential system, Abstr. Appl. Anal., 2013 (2013), 7 pages. 1

[24] P.-G. Wang, P. Li, Kth Order convergence for a semilinear elliptic boundary value problem in the divergence form, Appl. Math. Comput., 217 (2011), 8547-8551. 1

[25] P.-G. Wang, X. Liu, A periodic boundary value problem for nonlinear singular differential systems with "maxim", Bound. Value Probl., 2015 (2015), 18 pages. 1

[26] P.-G. Wang, X. Liu, The quadratic convergence of approximate solutions for singular difference systems with "maxima", J. Math. Computer Sci., 16 (2016), 227-238. 1

[27] P.-G. Wang, H.-X. Wu, Y.-H. Wu, Higher even-order convergence and coupled solutions for second-order boundary value problems on time scales, Comput. Math. Appl., 55 (2008), 1693-1705. 1 